\title{
Size effect for achieving high mechanical performance body-centered cubic metals and alloys
}

\author{
Yan $\mathrm{Lu}^{1}$, Xinyu Shu ${ }^{1,2}$ and Xiaozhou Liao ${ }^{3 *}$
}

\begin{abstract}
Submicron and nanostructured body-centered cubic (BCC) metals exhibit unusual mechanical performance compared to their bulk coarse-grained counterparts, including high yield strength and outstanding ductility. These properties are important for their applications in micro-, nano- and even atomic-scale devices as well as for their usages as components for enhancing the performances of structural materials. One aspect of the unusual mechanical properties of small-sized BCC metals is closely related to their dimensional confinement. Decreasing the dimensions of single crystalline metals or the grain sizes of polycrystalline metals contributes significantly to the strengthening of the small-sized BCC metals. In the last decade, significant progress has been achieved in understanding the plasticity and deformation behaviors of small-sized BCC metals. This paper aims to provide a comprehensive review on the current understanding of size effects on the plasticity and deformation mechanisms of small-sized BCC metals. The techniques used for in situ characterization of the deformation behavior and mechanical properties of small-sized samples are also presented.
\end{abstract}

Keywords: body-centered cubic metals, size effect, plastic deformation, mechanical properties

\section{INTRODUCTION}

Both the volume fractions of surface (VFS, which is determined by the sizes of single crystalline materials) and grain boundaries (VFGBs, which is determined by the grain sizes in polycrystalline materials) of materials are important in determining their mechanical behaviors and their applications. Small sizes of materials are important for applications in micro- and nano-technology [1,2] because of not only less consumption of materials (and therefore lower manufacturing cost) and reduced occupation space but also the unusual properties at small scale. Recent studies on small-sized materials with micro- or nano-dimensions revealed a variety of excellent properties compared with their bulk counterparts, including prominent mechanical properties $[3,4]$ and unique electronic properties $[1,2,5]$. These properties are related to the microstructural defects and the surface/ interfacial effects on electronic structures in micro- or nano-scaled materials. The VFGB of materials mainly concerns with the applications of structural materials [611]. Based on the well-known Hall-Petch relationship $[12,13]$, decreasing the grain size of metallic materials can enhance the strength of materials, which is caused by increasing the opportunities of interactions between dislocations and grain boundaries [14-17]. Therefore, significant efforts have been paid to improving the ability of engineering nanocrystalline metallic materials in order to strengthen materials $[18,19]$.

The mechanical properties of small-sized (both VFS and VFGB) materials have significant impacts on the stability of long-term performance of the materials and thus have drawn worldwide research interests. Some small-sized materials present superior mechanical properties, including ultra-large ductility [6-9] and ultra-large elasticity $[10,11,14-16,20,21]$, which are distinctive from their bulk counterparts and have been attributed to their size-dependent deformation mechanisms [17-19]. The change of material dimensions from bulk to micron or nano influences the volume fraction of internal microstructural defects and the surface effects on materials' mechanical behavior. Extensive experiments and computational simulations have revealed significant size effects on the plastic deformation mechanisms of materials [19,22-26]. For single crystalline materials, a "smaller is stronger" phenomenon exists in different crystal systems [27-33]. Various explanations were proposed to elucidate

\footnotetext{
${ }^{1}$ Beijing Key Lab and Institute of Microstructure and Properties of Advanced Materials, Beijing University of Technology, Beijing 100124, China

${ }^{2}$ Department of Material Science and Engineering, National University of Singapore, Singapore 117575, Singapore

${ }^{3}$ School of Aerospace, Mechanical and Mechatronic Engineering, The University of Sydney, Sydney, New South Wales 2006, Australia

* Corresponding author (xiaozhou.liao@sydney.edu.au)
} 
such size-dependent strengthening effect, including the dislocation starvation mechanism [34], the single-arm dislocation source mechanism and the dislocation source truncation mechanism [35] as well as the dislocation saturation effect [36]. Due to the high strength of smallsized single crystalline materials, it is possible to have dislocation nucleation and slip events in small-sized materials that are extremely brittle in their bulk form $[6,20,37,38]$. This would lead to outstanding plasticity in the small-sized materials. For example, $\mathrm{Si}$ and $\mathrm{SiC}$ nanowires experience dislocation nucleation and slip, and amorphization processes, leading to unusual superplasticity [7-9,39]. In polycrystalline metals, the HallPetch relationship applies that result in strengthening with reducing grain size before the grain size reaches a threshold value where an inverse Hall-Petch relationship takes effect [24-26,40,41]. Such transition lies in the crossover in plastic deformation mechanisms, from dislocation-mediated plasticity to GB-mediated plasticity.

The size effects on the elasticity and plasticity of facecentered cubic (FCC) materials, which are the most frequently used materials in micro- and nano-technology, have been extensively investigated [22-26,29,32-36,40,41] and comprehensively reviewed [3,4,42-45]. Recently, body-centered cubic (BCC) metals have triggered increasing interest for their broad applications [46-51]. However, there has been no systematic review on the size effect on their plasticity. This review will cover the studies of size effect on the plasticity in both single crystalline and polycrystalline BCC metals. As in situ straining in the electron microscope has been the most important methods for the investigations of the atomic-scale mechanisms of plasticity of small-sized materials [2733], the most frequently used in situ straining experimental techniques will be briefly introduced. Then the fundamental of dislocation-mediated and GB-mediated plasticity in BCC metals will be introduced that provides the background. The subsequent discussion of the size effect on plasticity in BCC metals will be divided into two parts with or without internal planar defects. The size effect on the plasticity of BCC single crystalline metals, including stress responses, plastic morphology and plastic deformation mechanisms, and temperature dependence of the size effect will be addressed respectively in two sections. Afterwards, the grain size effect on the plasticity of BCC polycrystalline metals will be addressed. Finally, the conclusion remarks and outlook will be presented.

\section{EXPERIMENTAL TECHNIQUES}

The mechanical properties of bulk materials are tested using conventional methods [52] in which the materials sample sizes are usually in the millimeter and centimeter scales $[53,54]$. When sample dimensions reduce to microscale or smaller, a special platform is needed for viewing and manipulating the small samples. For years, in situ straining microscopy techniques that allow simultaneous straining (including mechanical testing) and microstructural characterization have been developed for the investigations of the plasticity of small-sized materials. The most popular platforms for these in situ techniques are the scanning electron microscopy (SEM) and the transmission electron microscopy (TEM) that can be used to reveal the mechanisms of the plastic deformation of small-sized samples at the nanometer and sub-nanometer resolution, respectively.

Many commercial devices have been developed for in situ uniaxial mechanical straining in SEM, including MTS NanoXP system [28,55], Hysitron SEM PicoIndenter [56], Nanomechanic InSEM [57], and some custom-built devices (such as SEMentor [58-60]). These devices have been used to reveal the size effects on the plasticity and deformation mechanisms of sub-micro-scaled BCC metals, which will be discussed in section of THE SIZE EFFECT ON THE PLASTICITY OF SMALL-SIZED BCC SINGLE CRYSTALLINE METALS and THE TEMPERATURE DEPENDENCE OF THE SIZE EFFECT ON SINGLE CRYSTALLINE BCC METALS. While stressstrain curves obtained from in situ testing tell the mechanical properties of micro- and nano-sized samples, SEM observations of the evolution of surface morphology of samples during deformation processes provide important information on the size effect on deformation mechanisms. Unfortunately, SEM observations do not provide information on the deformation processes inside materials. To observe the processes of deformationinduced dislocation nucleation and gliding, deformation twinning, grain boundary activities, and the interactions between different defects, in situ straining in TEM is essential. Available equipment for in situ straining in TEM includes the Gatan straining holder [61], Hysitron TEM PicoIndenter [56], and Nanofactory STM-TEM holder [62].

The working mechanisms of all straining devices for in situ experiments in both SEM and TEM are similar. Fig. 1 presents schematics of compression of a pillar [28,55,5760] (Fig. 1a), tension of a dog-bone-shaped sample [58] (Fig. 1b) fabricated from bulk materials using the focused-ion-beam (FIB) technique, and compression and tension $[56,61,62]$ of as-grown nanowires (Fig. 1c and d), respectively. In compression tests, the pillars and 

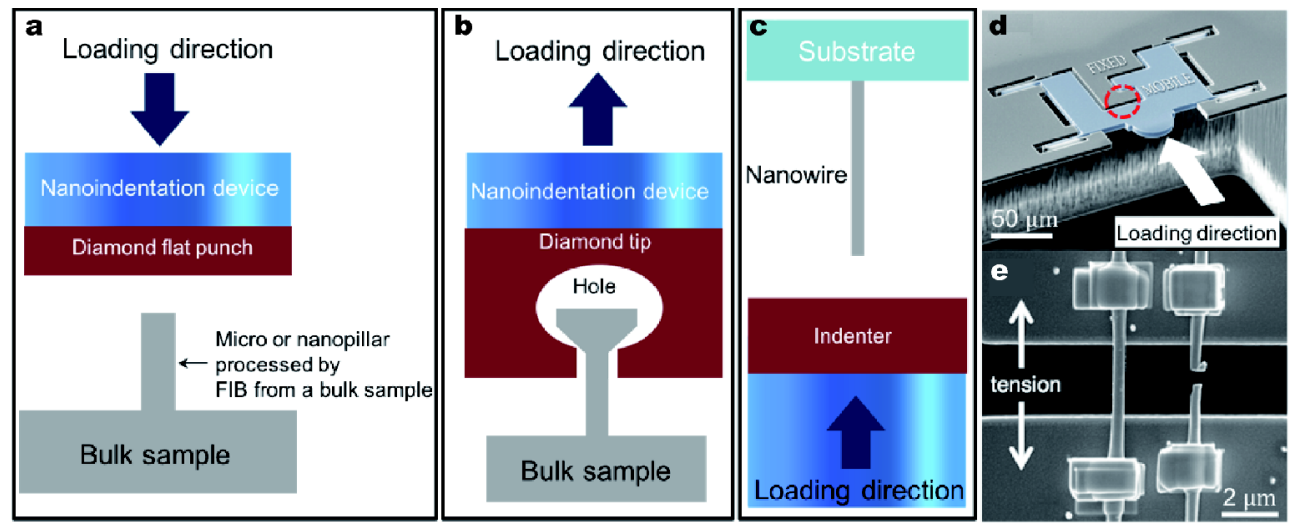

Figure 1 The schematics of compression and tension of FIB-processed pillars and as-grown nanowires. All the indenters can be manually controlled. (a) Compression of a micro- or nanopillar. (b) Tension of a micro- or nanopillar. A hole and a thin slit are ion-etched in order to grip the dog-boneshaped sample. (c) Compression of an as-grown nanowire. The nanowire is fixed on the substrate. (d, e) SEM images of PTP device with two nanowires welded on [56,63]. Reprinted from Ref. [63], Copyright 2012, Elsevier.

nanowires are compressed with a flat punch usually made from a diamond tip; the punch can be manually controlled to determine the loading/unloading direction and compression depth. Devices for tensile tests are slightly complicated. A hole and a thin slit are ion-etched on the diamond tip in order to grip dog-bone-shaped samples for tensile testing, as shown in Fig. 1b. For the asgrown nanowires, the push-to-pull (PTP) device from Hysitron provides a convenient way to convert pushing force into pulling force for tensile testing, as shown in Fig. $1 \mathrm{~d}$ and e [56,63]. The mobile part of the PTP device is connected to the fixed part via four arms. A nanowire bridges the gap between the mobile and the fixed platforms. The two ends of the sample are welded on the mobile and fixed platforms, respectively. When the flat punch is pushed forward, a mechanical pulling stress (tension) is loaded on the sample.

Unfortunately, most commercially available facilities for in situ straining in TEM only allow single-tilt operation of specimens, making it extremely difficult to image structures at the atomic resolution during the deformation process [64]. The problem was overcome by Han and his colleagues. They conducted in situ atomicscale TEM investigations of structural evolution under loading using a Gatan double-tilt heating holder and a thermal-activated bimetal device, as shown in Fig. 2a-c. The method can be used for both tension and indentation tests of samples in various forms including thin films, nanowires and FIB-processed samples. Fig. 2b, $c$ present the schematics of tensile and indentation experiments, in which the yellow strips stand for the thermal-activated actuators and they can bend outwards for tension and

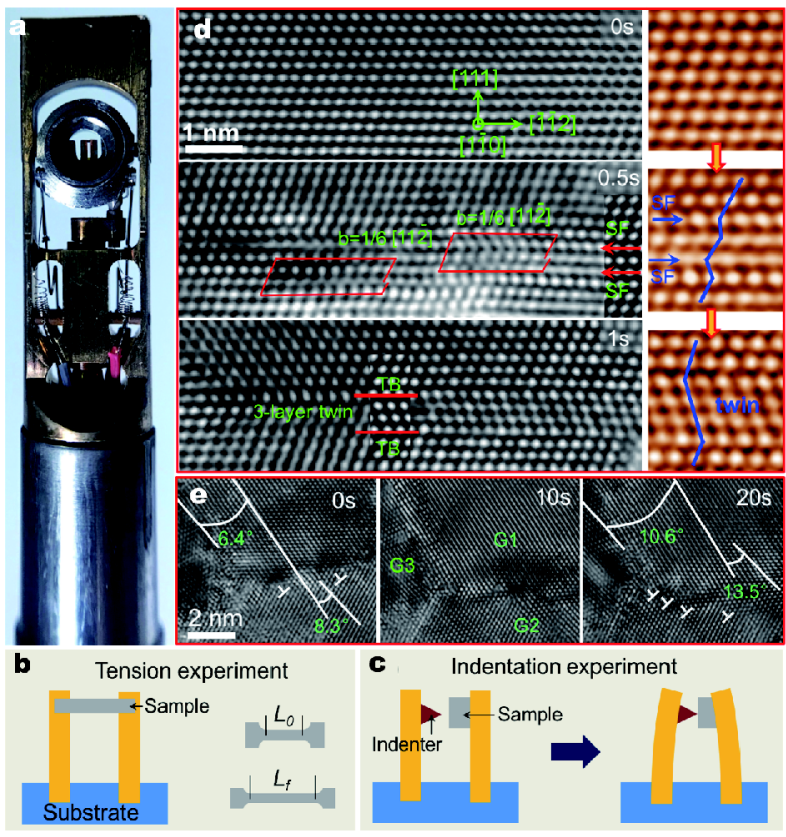

Figure 2 (a) A Gatan double-tilt heating holder with a bimetal tension device installed. (b, c) Schematics of tension and indentation devices. The yellow strips are thermal-activated actuators. Their freestanding end can bend outwards for tension (b) and inwards for indentation (c). (d) In situ TEM observation of a deformation twinning process in a nanocrystalline Pt film, reproduced from Ref. [65], Copyright 2017, Nature Publishing Group. (e) In situ TEM observation of grain rotation in a nanocrystalline Pt film, reproduced from Ref. [25], Copyright 2014, Nature Publishing Group.

inwards for indentation [14,15]. Fig. 2d, e exemplify the atomic-resolution TEM images of deformation twinning [65] and grain rotation [25] processes in a nanocrystalline Pt film obtained using the technique. 


\section{PLASTIC DEFORMATION MECHANISMS OF BCC CRYSTALLINE STRUCTURES}

Plastic deformation of BCC crystalline structures is accommodated by a series of activities of crystalline defects. Crystalline defects are the periodicity of crystalline structures stops. They include linear defects (dislocations) and planar defects like stacking faults, twin boundaries, grain boundaries, and surface of materials. Crystalline defects formed during the synthesis and processing processes of materials are affected significantly by external fields (such as stress field and electric field) [1-5]. They influence significantly the physical and mechanical properties of materials. A brief overview of crystalline defects in BCC structures will be introduced in this section. Details of dislocations and grain boundaries in BCC crystalline structures will be discussed, which is important for the subsequent discussion of the plasticity of BCC crystalline structures in next two sections.

\section{Dislocation-mediated plasticity in BCC crystalline structures}

Plastic deformation of crystalline metallic materials can be achieved by several mechanisms. The most important mechanism in coarse-grained crystalline metallic materials is dislocation slip [66]. When a dislocation glides through a grain and disappears at a grain boundary or the surface of the material, plastic deformation occurs with the amount of the plastic displacement being equal to the length of the Burgers vector of the dislocation and the direction of the displacement being the same as that of the Burgers vector. Large plastic deformation can be the effect of the slip of a large number of dislocations. Dislocations can be classified into full dislocations and partial dislocations depending on the magnitude of their Burgers vectors. The magnitude of the Burgers vector of a full dislocation is an integral multiple of a unit lattice length, while that of a partial dislocation is a fractional multiple of a unit lattice length. Dislocations can only glide on slip planes along certain slip directions. A slip plane and a slip direction (the direction of the Burgers vector) form a slip system. Slip systems vary significantly with crystalline structures. Slip normally occurs on closepacked crystalline planes along close-packed directions for energy minimization [17]. Sometimes slip can also occurs on non-close-packed planes due to structural complexity, dislocation reactions and/or extreme external conditions like high stress and/or high temperature [17]. A dislocation with its Burgers vector lying on a slip plane is a mobile or glissile dislocation, while a dislocation with its Burgers vector not lying on any slip plane is an immobile or sessile dislocation.

Depending on the geometrical orientation relationship between a dislocation line and its Burgers vector, part of a dislocation can be edge-type where the dislocation line is perpendicular to its Burgers vector, screw-type where the dislocation line is parallel to its Burgers vector, or a mixed-type in which the angle between the dislocation line and its Burgers vector is of any value between $0^{\circ}$ to $90^{\circ}$. Different parts of a dislocation line can have different types depending on the local orientation of the dislocation line. However, there is only one Burgers vector of a dislocation line. Computational and experimental investigations have confirmed that the Burgers vectors of most glissile full dislocations in BCC metals are usually $1 / 2<111>$, where $<111>$ are the close-packed directions. Sometimes dislocations with a Burgers vector of $<100>$ formed through the interaction of two $1 / 2<111>$ dislocations $[17,66-71]$ :

$$
\frac{1}{2}\langle 11 \overline{1}\rangle+\frac{1}{2}\langle 1 \overline{11}\rangle \rightarrow\langle 100\rangle .
$$

There are many slip planes for full dislocations in BCC metals [72-74]. Many factors affect the activation of slip systems. These include temperature, the direction of applied stress relative to the crystallographic orientation of a grain, sample dimensions, and the material itself. A systematic review on slip planes in BCC metals activated at different temperatures is available [54]. In most cases, slip occurs on $\{110\}$ and $\{112\}$ planes. For example, it is $\{110\}$ in pure $\mathrm{Fe}$ at the ambient and low temperature $[73,74]$, both $\{110\}$ and $\{112\}$ in pure Mo [75,76], and only $\{112\}$ for pure $V$ [75]. Occasionally, other slip planes, such as $\{123\}$ and other crystalline planes with high index $\{h k l\}$ were also reported $[66,73,74,77,78]$.

It is very important to note that the plastic deformation of BCC crystalline metals is controlled by the motion of screw dislocations due to their non-planar core structure $[28,44,69,71]$. In BCC metals, three $\{110\}$ planes and three $\{112\}$ planes belong to a same $<111>$ zone. A $1 / 2<111>$ screw dislocation would dissociate on these slip planes, generating the non-planar core structure $[79,80]$. Two kinds of core structures have been proposed [71,81-83]: the degenerate dislocation core structure (three-fold structure) and the non-degenerate dislocation core structure (six-fold structure), as shown in Fig. 3a. These non-planar core structures contribute to two well-known characteristics of BCC metals: a) the breakdown of Schmid law so that slip can occur on a crystallographic plane not possessing the maximum resolved shear stress $[44,71]$, and b) strong temperature-dependent mobility of 

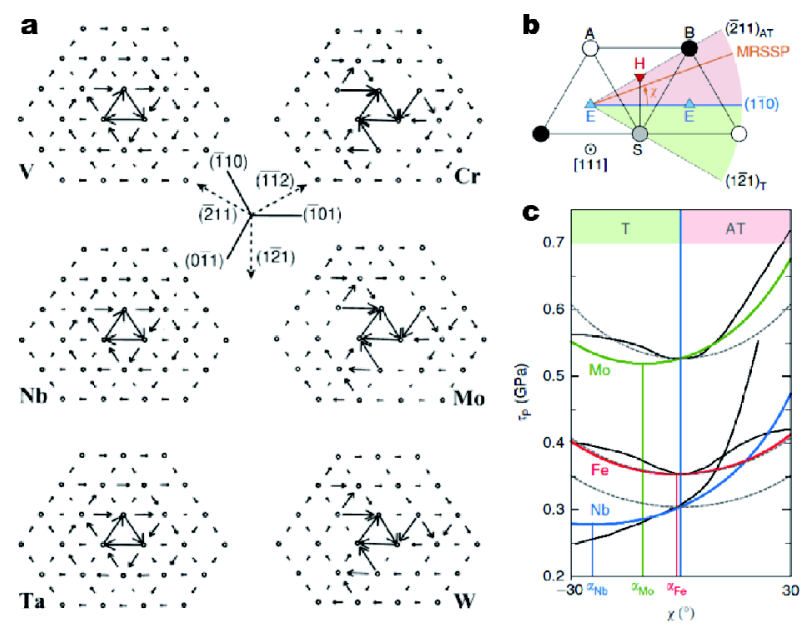

Figure 3 Core structures of screw dislocations in BCC metals. (a) The six-fold structure of $\mathrm{V}, \mathrm{Nb}$, and $\mathrm{Ta}$, and the three-fold structure of $\mathrm{Cr}$, Mo, and W, reproduced from Ref. [71], Copyright 1998, Elsevier. (b, c) Twinning-antitwinning asymmertry indication, reproduced from Ref. [25], Copyright 2014, Nature Publishing Group.

screw dislocations [28].

The non-Schmid behavior of BCC metals has two implications [71]. First, the critical resolved shear stress (CRSS) will change with the slip system and the relative direction of slip. This phenomenon can lead to the twinning-antitwinning asymmetry in BCC metals under external stresses, exhibiting an anisotropic compressiontension stress response, as shown in Fig. 3b, c [83]. Several theoretical studies show the details of this anisotropy in the plasticity of BCC metals as presented in Fig. 3b, c [71,81-83]. Second, CRSS can be influenced by non-glide component of the applied stress tensor. Concerning this aspect, it was [71] concluded that those non-glide components of the applied stress tensor can interact with small edge dislocation components in the screw dislocation core, leading to the change in the dislocation core structure that increases the CRSS. Due to the increase of CRSS for screw dislocations, which also implies the increase Peierls stress for screw dislocations, the mobility of screw dislocations or the screw component of mixed dislocations is much lower than that of edge dislocations or the edge component of mixed dislocations [44].

It should be noted that the mobility of screw dislocations has a strong correlation with the stress response of BCC metals $[28,84,85]$. Low mobility of screw dislocations requires large external stress to activate, corresponding to large stress response. Besides of external stress, increasing temperature improves the mobility of screw dislocations and thereby reduces the external stress
Table 1 Critical temperature $T_{\mathrm{c}}$ of some BCC metals

\begin{tabular}{cc}
\hline BCC metals & Critical temperature (K) \\
\hline $\mathrm{W}$ & $800 \mathrm{~K}[99], 760 \mathrm{~K}[85]$ \\
$\mathrm{Mo}$ & $450 \mathrm{~K}[28], 472 \mathrm{~K} \mathrm{[85]}$ \\
$\mathrm{Ta}$ & $450 \mathrm{~K}[99], 440 \mathrm{~K}[85]$ \\
$\mathrm{Nb}$ & $290 \mathrm{~K}[85], 350 \mathrm{~K}[28]$ \\
$\mathrm{V}$ & $380 \mathrm{~K}[107]$ \\
$\alpha-\mathrm{Fe}$ & $340 \mathrm{~K}[108]$ \\
\hline
\end{tabular}

needed to activate the dislocations. A critical temperature $\left(T_{\mathrm{c}}\right)$, which is defined as the temperature at which the flow stress becomes insensitive to the test temperature and screw and edge dislocations have the same mobility, has been proposed as an intrinsic feature of BCC metals [28]. Below $T_{c}$, screw dislocations have low mobility due to the lack of thermal activation. Therefore, large external stresses are necessary to sustain plastic deformation in BCC metals at low temperatures. Table 1 lists the critical temperature of some BCC metals.

A full dislocation can dissociate into several partial dislocations. A $1 / 2<111>$ dislocation can dissociate on one $\{110\}$ plane or one $\{112\}$ plane [86-88], in the ways presented below:

$$
\begin{gathered}
\frac{1}{2}\langle 111\rangle=\frac{1}{8}\langle 110\rangle+\frac{1}{4}\langle 112\rangle+\frac{1}{8}\langle 110\rangle, \\
\frac{1}{2}\langle 111\rangle=\frac{1}{6}\langle 111\rangle+\frac{1}{3}\langle 111\rangle .
\end{gathered}
$$

A $1 / 2<111>$ dislocation can also dissociate on three intersecting $\{110\}$ planes or two intersecting $\{112\}$ planes in the ways presented below, respectively [88]:

$$
\begin{aligned}
& \frac{1}{2}\langle 111\rangle=\frac{1}{8}\langle 110\rangle+\frac{1}{8}\langle 101\rangle+\frac{1}{8}\langle 011\rangle, \\
& \frac{1}{2}\langle 111\rangle=\frac{1}{6}\langle 111\rangle+\frac{1}{6}\langle 111\rangle+\frac{1}{6}\langle 111\rangle .
\end{aligned}
$$

The $\{110\}$ planes in BCC crystals can be seen as in a stacking sequence of "ABABAB...". Atoms can move to interstitial sites to form a partial dislocation with a Burgers vector of $1 / 8<110>$ (see Fig. $4 a)$. The $\{112\}$ planes are the coherent twinning planes in BCC crystals that arrange in an order of "ABCDEFABCD..." (Fig. 4b). Partial dislocations with each one on a consecutive $\{112\}$ plane and with the Burgers vector of $1 / 6<111>$ or $-1 / 3<111>$ can form a crystal twin, with a twin thickness at least of three atomic layers [89].

The dislocation theory discussed above is based on bulk single crystalline and bulk coarse-grained BCC materials. With the decrease of sample/grain size, the type of dislocations controlling plastic deformation processes 

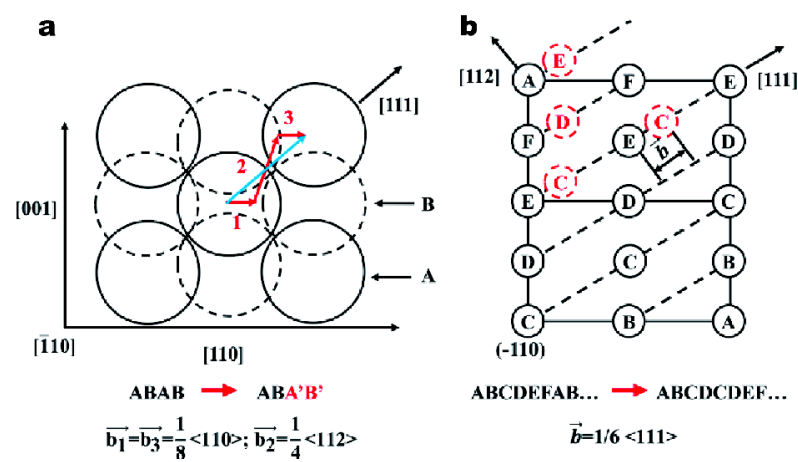

Figure 4 Schematics of two types of partial dislocations in BCC crystals. (a) A full dislocation with a Burgers vector of $1 / 2<111>$ (as presented by a blue arrow) composes two $1 / 8<110>$ partial dislocation (presented by red arrows 1 and 3 ) and one $1 / 4<112>$ partial dislocation (presented by red arrow 2). The end points of arrows 1 and 2 indicate the interstitial positions on $\{110\}$ plane of BCC crystals. (b) The $1 / 6<111>$ partial dislocation leads to a crystal twin with a thickness of three atomic layers.

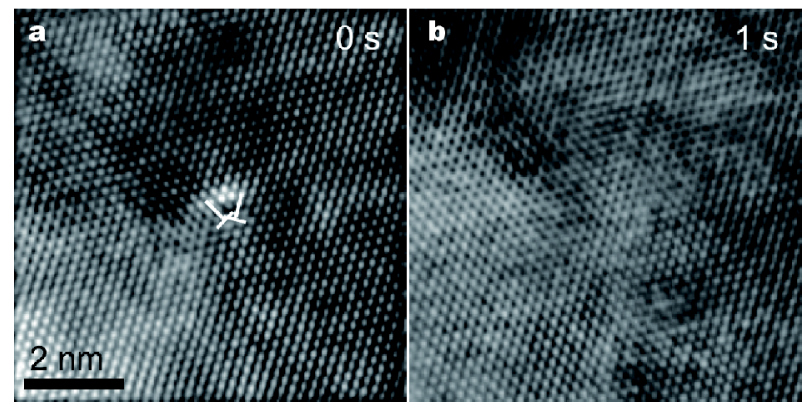

Figure 5 In situ TEM observation along a $<111>$ direction of a single crystalline Mo nanowire under tensile loading. A mixed type dislocation with its edge component seen in (a) glided rapidly out of the region in one second, leaving behind a perfect crystalline area, as shown in (b) (unpublished).

would change. In single crystalline Mo nanowires, plenty of edge dislocations or dislocations with significant edge components were observed during in situ tensile deformation. Fig. 5 presents an example showing the nucleation and motion of an edge component from a mixed type dislocation in a Mo nanowire observed from a $<111>$ zone axis (unpublished work). The dislocation in Fig. 5a glides away from this region within one second under tensile loading, leaving behind a perfect crystalline area as shown in Fig. 5b. Further investigation is needed to understand the critical size for the transition of dislocation type and the contribution of edge dislocations to the total plastic deformation at the small size scale.

\section{GB-mediated plasticity in BCC metals}

Reducing grain size increases the VFGB of materials. GBs can be heavily involved in the plastic deformation processes of materials and their contribution to the plastic deformation depends on grain size. Previous investigations of polycrystalline FCC metals show that the competition between GB-mediated plasticity and dislocation-mediated plasticity determines the transition from a Hall-Petch relationship to an inverse Hall-Petch relationship [22-25]. This transition also exists in BCC metals [90-94]. GBs activities in BCC metals are quite similar to those in FCC metals. They comprise GB sliding (atoms at both sides of a GB move in opposite directions parallel to the GB), grain rotation (a grain rotates around an out-of-plane axis) and grain tilting (a grain rotates around an in-plane axis) [90-92]. GBs can also act as sources and sinks of dislocations. Simulation results [9397] suggest that these GB activities strongly depend on factors including shear direction, temperature and GB structure.

\section{SIZE EFFECT ON THE PLASTICITY OF SMALL-SIZED BCC SINGLE CRYSTALLINE METALS}

The size-dependent plasticity of small-sized BCC metals has been revealed by uniaxial mechanical tests [58-60]. Compared to FCC metals, BCC metals present more complicated size dependency of plasticity. Previous studies indicate that the size effect on the plasticity of BCC metals lies in many aspects, including stress responses, plastic morphology, and plastic deformation mechanisms.

\section{Size-dependent yield strength and flow stress}

Yield strength and flow stress inversely scale with sample size in single crystalline metals, which is referred to as "smaller is stronger" $[20,48,58,59,89-94,98-101]$ and can be described using the following equation:

$$
\sigma_{y}=\sigma_{0}+k d^{-n},
$$

where $\sigma_{y}$ is yield strength, $\sigma_{0}$ is bulk stress of materials that is usually referred as lattice resistance and is directly dependent on temperature, $d$ is the sample size (grain size for polycrystalline materials and diameter for single crystalline materials), and $k$ and $n$ are constants. The power-law exponent $n$ represents the sensitivity of stress responses to sample size, which has been confirmed in the order of 0.5 to 1 in FCC single crystalline metals $[30,44,60]$. For BCC single crystalline metals, $n$ varies significantly for different metals due to their distinctive properties and test temperatures, which will be discussed in detail in the section of "The temperature effect on size 

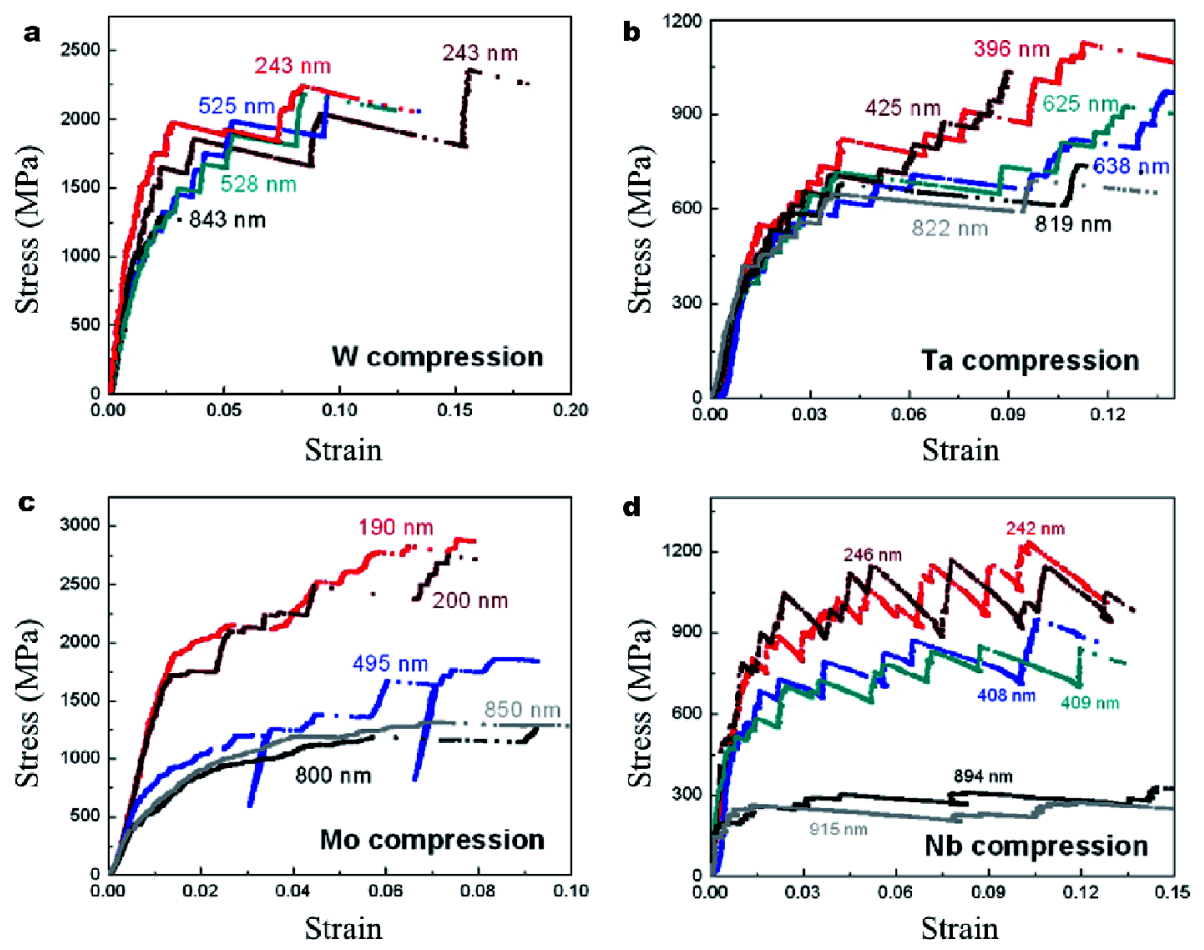

Figure 6 Uniaxial compression on BCC single crystalline W, Mo, Nb, and Ta samples at the same strain rate and temperature, reproduced from Ref. [85], Copyright 2010, Elsevier.

strengthening exponent $n$ ". The flow stress of a crystal is composed of two main components: the long-range elastic interactions of mobile dislocations and the stress needed for dislocations to overcome local energy barriers for slip (lattice resistance).

Uniaxial compression tests of BCC metals $\mathrm{W}, \mathrm{Mo}, \mathrm{Nb}$, and $\mathrm{Ta}$, as shown in Fig. 6, demonstrate clear size effects on their mechanical responses [85]. There are two prominent size-dependent properties presented by the compressive stress-strain curves in Fig. 6. (1) the plastic deformation regions in these metals with small diameters $d(d<500 \mathrm{~nm})$ present discrete strain bursts, which are instantaneous stress drops with small increase of strain, followed by linear reloading segments. This is in contrast to the smooth stress-strain curves of samples with large diameters $(d>500 \mathrm{~nm})$ that presented steady work hardening processes. Theoretical [101] and experimental $[102,103]$ studies confirm that such unusual mechanical responses in small-sized BCC metals are attributed to dislocation avalanche. Dislocations can interact with each other and get into mutually trapped configurations. The long-range mutual interactions between dislocations in small-sized samples can destruct the mutually trapped configurations and lead to avalanche-like dislocation motion, presenting a stress slightly drop with increasing strain. The avalanche strains are inversely proportional to sample size. Therefore, it is difficult to observe strain bursts in samples with large sizes. Instead of dislocation avalanche, samples with large sizes favor continuous work hardening and thereby present continuous strain processes. (2) the yield stress and flow stresses in $\mathrm{W}, \mathrm{Nb}, \mathrm{Mo}$, $\mathrm{Ta}$ are consistent with the "smaller is stronger" phenomenon.

The size effect on strengthening can be explained by two main mechanisms for BCC metals: the weakest-link mechanism $[99,104,105]$ and the dislocation starvation mechanism $[27,84,86]$. The weakest-link mechanism emphasizes the dislocation effect on strengthening of small-sized metals. It is believed that the length of dislocation sources in metals determines the sizedependent stress responses and that the stress needed to activate a dislocation source is inversely proportional to the length of the dislocation source $[104,105]$. The length of dislocation sources is short in small-sized metals due to the confinement of the small sample volume. Large stresses are therefore expected to activate short dislocation sources in small samples to sustain plasticity. For BCC metals, plasticity is usually governed by screw dislocations. The cross-slip of screw dislocations increases the opportunities of dislocation interactions that can 
possibly form sessile junctions. Dislocation slip can be hindered by these junctions, which increases the dislocation density in the materials. Increasing the dislocation density can truncate dislocations and thus reduce the length of dislocation sources, contributing to large yield and flow stresses and thereby assisting weakest-link strengthening. Dislocation starvation is the mechanism of mechanical annealing. External stress can drive preexisting dislocations out of samples, leading to dislocation starvation. Due to exhaustion of dislocations and dislocation sources inside small-sized metals, a higher stress is expected to promote the nucleation of dislocations on surface to sustain further plasticity. It is clearly proposed that metallic materials with small sizes can assist such driving process due to larger image stress (caused by large specific surface) and shorter distance (because of small volume) for dislocation escape from surface before entanglement [28,59]. Therefore, the "smaller is stronger" can possibly arise from dislocation starvation.

Theoretically, a transition from the weakest-link mechanism to the dislocation starvation mechanism can occur at a certain sample size. The weakest-link mechanism is believed only applicable to those with sizes larger than hundreds of nanometers [105], while dislocation starvation occurs in samples with sizes at nanoscale. However, a high density of tangled dislocations was observed in compressed Mo pillars even with a diameter of $\sim 100 \mathrm{~nm}$ [85]. As mentioned before, dislocation interactions can result in a high density of dislocations in metals, leading to shorter dislocation source lengths and thereby larger flow stresses, and therefore, Mo pillars with diameters of $\sim 100 \mathrm{~nm}$ was strengthened by the weakest-link mechanism. This seems contradictory to the frequently observed dislocation starvation mechanism in BCC metallic pillars with diameters of hundreds of nanometers as shown in Fig. 7 [63]. The explanation for the high densities of dislocations in plastically deformed BCC metals with such small dimensions $(\sim 100 \mathrm{~nm}$ in diameter) was proposed that dislocation starvation exists at a certain stress range throughout the test, while dislocation density starts to increase under higher loading stress. When the stress was kept at a relative high value, dislocations nucleated continuously and moved fast, leading to a steady state of dislocation density and consequently large strain [31]. Fig. 8 shows ultra-large elongation of a single crystalline Mo nanowire with a diameter of $130 \mathrm{~nm}$. Under higher loading stress, the increased density of dislocations could arise from dislocation self-multiplication through cross-slip

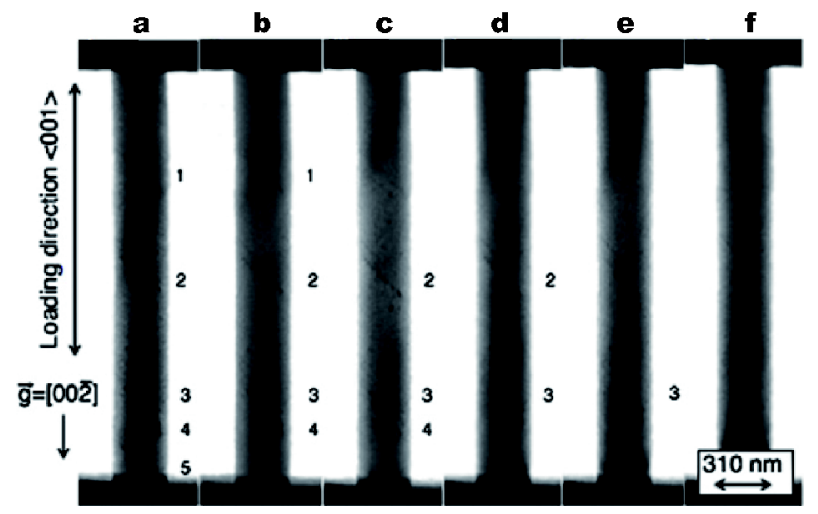

Figure 7 A series of images showing a dislocation starvation process of a Mo alloy nanofiber during tensile deformation. Numbers 1-5 label dislocations that were introduced by $9 \%$ pre-straining. The number of dislocations decreased with loading, reproduced from Ref. [63], Copyright 2010, Elsevier.

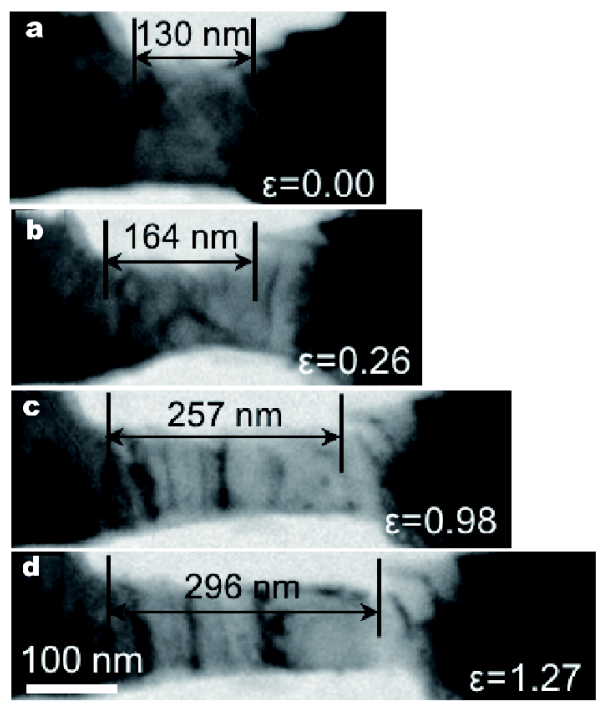

Figure 8 Ultra-large plastic deformation of a single crystalline Mo nanowire with a diameter of $130 \mathrm{~nm}$. The homogeneous elongation reached $127 \%$, reproduced from Ref. [31], Copyright 2016, Nature Publishing Group.

[100,106-108] in BCC metals. Therefore, except for sample size, the applied stress is another factor determining deformation mechanisms. Different mechanisms would operate at different deformation stages. For example, as shown in Fig. 9, a compression test of Mo nanopillar, dislocations are emitted from surface at the initially low stress regime (Fig. 9a, b). Subsequent compression leads to dislocation starvation, which is also called mechanical annealing, at later stages (Fig. 9c, d) of the deformation and dislocation self-multiplications at high stress levels (from Fig. 9d to e) [100]. 


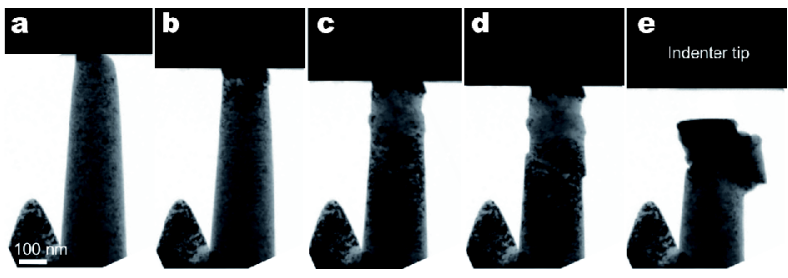

Figure 9 Dislocation starvation (mechanical annealing) during the in situ compression test of a single crystalline Mo nanopillar with a diameter of $142 \mathrm{~nm}$ at the free end, reproduced from Ref. [100], Copyright 2011, Nature Publishing Group.

Another important factor influencing the crossover between two strengthening mechanisms is the preexisting dislocation density before straining. Chisholm et al. [63] emphasized on the effect of the pre-existing dislocation density on the dislocation starvation mechanism through uniaxial tension test of a BCC Mo alloy. A Mo alloy nanowire with a medium pre-existing dislocation density $\left(<10^{14} \mathrm{~m}^{-2}\right)$ presents dislocation starvation during tensile loading (Fig. 7), while that with a high dislocation density $\left(\geq 10^{14} \mathrm{~m}^{-2}\right)$ is accompanied by a high density of dislocations throughout the whole test.

Previous studies confirm that the weakest-link mechanism demonstrates a positive effect of dislocation density on yield strength $[104,105]$. A high density of dislocation leads to an increase in work hardening and thereby a large CRSS. On the contrary, there are many experiments showing opposite results $[63,102,103]$. These studies confirm that a high dislocation density in smallsized BCC metallic pillars always leads to low flow stress and yield strength. For materials with high dislocation densities, dislocations do not have to nucleate on the surface or grain boundaries and the yield strength and flow stresses are determined by collective dislocation motion, dislocation multiplication, and dislocation interactions, corresponding to a more bulk-like continuous deformation. For materials with low dislocation densities, dislocations can be easily driven out by external stress, leading to dislocation starvation. The corresponding deformation usually starts with a sudden strain burst in a stress-strain curve, followed by higher stress and frequent strain bursts. Such contradictory that dislocation density leads to different stress responses, is attributed to different sample sizes. As mentioned before, the weakestlink model does not apply to small-sized samples. Increasing dislocation density in large samples (such as samples at the microscale) results in pronounced work hardening. When sample size decreases to the nanoscale, the strengthening mechanism transfers from weakest-link to dislocation starvation. Compared to small-sized

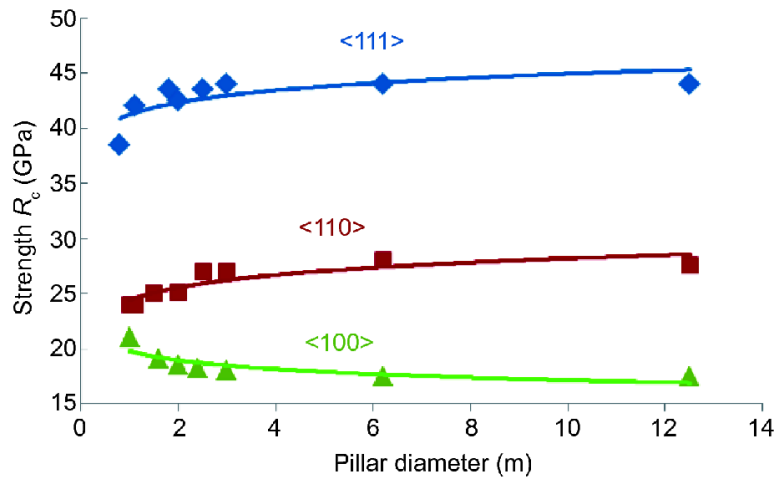

Figure 10 Size effect on yield strength of Mo nanowires at low temperature $(77 \mathrm{~K})$ for three crystallographic loading directions, reproduced from Ref. [109], Copyright 2015, Elsevier.

materials with high dislocation densities, small-sized materials with no or few dislocations can present nearly ideal strength under external stress. Therefore, dislocations density has a negative impact on the strength of small-sized materials.

The "smaller is stronger" is common in BCC single crystalline materials. However, recent simulations predict more complicated size dependence of stress responses of BCC single crystalline Mo nanowires at certain loading directions when the diameter is below $15 \mathrm{~nm}$ [109-111]. As shown in Fig. 10, uniaxial tensile tests of Mo nanowires with diameters smaller than $15 \mathrm{~nm}$ along different directions present distinguishing size effects on yield strength. For tensile tests along $<110>$ and $<111>$ directions, the yield strength is insensitive to diameter when the diameter is above $3 \mathrm{~nm}$; the yield strength decreases rapidly with decreasing diameter when the diameter is smaller than $3 \mathrm{~nm}$. For tensile tests along a $<100>$ direction, the yield strength is insensitive to diameter when the diameter is larger than $2 \mathrm{~nm}$, followed by a rapid increase with decreasing diameter to below $2 \mathrm{~nm}$. This complicated size dependence of yield strength arises from the significant role of free surface in yield strength and the plastic paths different from that in its large BCC counterparts. The approximation of yield strength for $\langle 110\rangle$ and $\langle 111\rangle$ tension tests is:

$$
\sigma_{y} \approx \frac{\tau_{c}}{m}-\frac{4 f_{x x} d}{h(d-h)}
$$

and for $<100>$ tension tests that is:

$$
\sigma_{y} \approx \frac{\tau_{c}}{m}-\frac{f_{x x} d}{(1+\beta) h(d-h)}+\frac{4 \beta f_{x x} d}{(1+\beta)\left(d-2 h^{*}\right)^{2}},
$$

where $\tau_{c}$ is critical stress of crystal, $f_{x x}$ is surface tension along the sample axis, $d$ is diameter of sample, $m$ is orientation factor for system where shear instability 
occurs, $h$ is the thickness of a layer where the distribution of local shear stresses is homogeneous, $\beta$ is the ratio of twin area located under the compressive stress to that under the tensile stresses, and $h^{*}$ is thickness of the layer in the compressed region. For Mo nanowires under $<110>$ and $<111>$ loading directions, full dislocationmediated plasticity dominates the whole plastic deformation. When $d \leq 4-5 \mathrm{~nm}$, the reduction of $d$ can result in a decrease in $\sigma_{y}$. For Mo nanowires tensile deformed along $<100\rangle$, deformation twinning occurs as the dominant plastic path, leading to the difference in stress state. Therefore, the stress increases with the reduction of $d$ when $d \leq 2 \mathrm{~nm}$.

\section{Size-dependent strain hardening rate}

Experiments confirm that the strain hardening rate (SHR) of BCC metals depends on sample size [99]. Fig. 11 summarizes compressive SHR data of BCC metals from previous work. Here, SHR is defined as the slope of a stress-strain curve between $2 \%$ and $5 \%$ strain. All those tests were conducted at the ambient temperature. SHRs of these BCC metals are inversely proportional to the diameter of samples. This size effect cannot be explained by the dislocation starvation mechanism because the increased SHR results from more significant work hardening that demands a certain dislocation density. Previous study [99] attributed such size effect to the weakest-link mechanism. It takes higher stress to activate a dislocation source with a shorter length and this leads to larger SHR in metals at a smaller scale. An obvious phenomenon in Fig. 11 is that the sensitivity of SHR to sample size varies with BCC metals. Such distinction arises from different critical temperatures $\left(T_{\mathrm{c}}\right)$ of various materials. According to Table 1, for example, W [85,99] has the highest $T_{c}$ that indicates the largest lattice resistance for screw dislocation motion than other BCC metals at the ambient temperature. Low mobility of screw dislocations in BCC metals contributes to more frequent dislocation interactions and cross-slip, leading to shorter lengths of dislocation sources and thereby larger size dependence of SHR.

\section{Size-dependent plastic deformation morphology}

Plastically deformed bulk BCC metals with sizes larger than hundreds of micrometers always show wavy morphologies at the side-walls (see Fig. 12a) due to their complicated dislocation slip systems and frequent crossslip of screw dislocations [112]. However, no wavy morphology is observed when the sizes of BCC metals decrease to the submicron-scale $[28,59,60,85,98]$. For

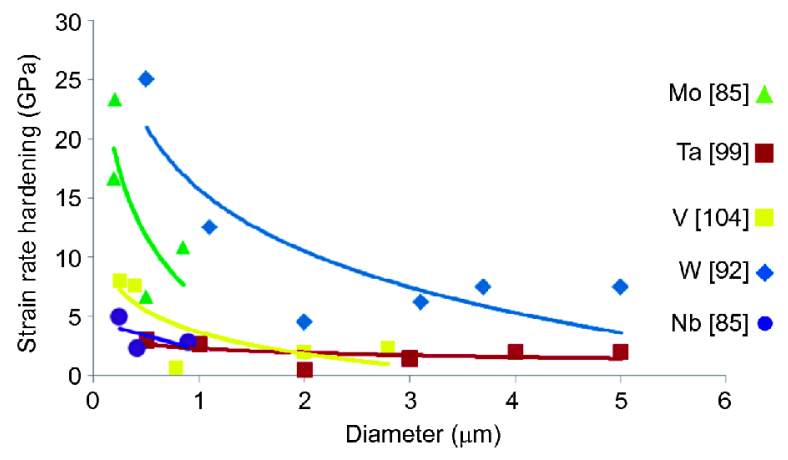

Figure 11 The dependence of strain rate hardening on sample diameter in single crystalline BCC metals $\mathrm{Mo}, \mathrm{Ta}, \mathrm{V}, \mathrm{W}$, and $\mathrm{Nb}$.

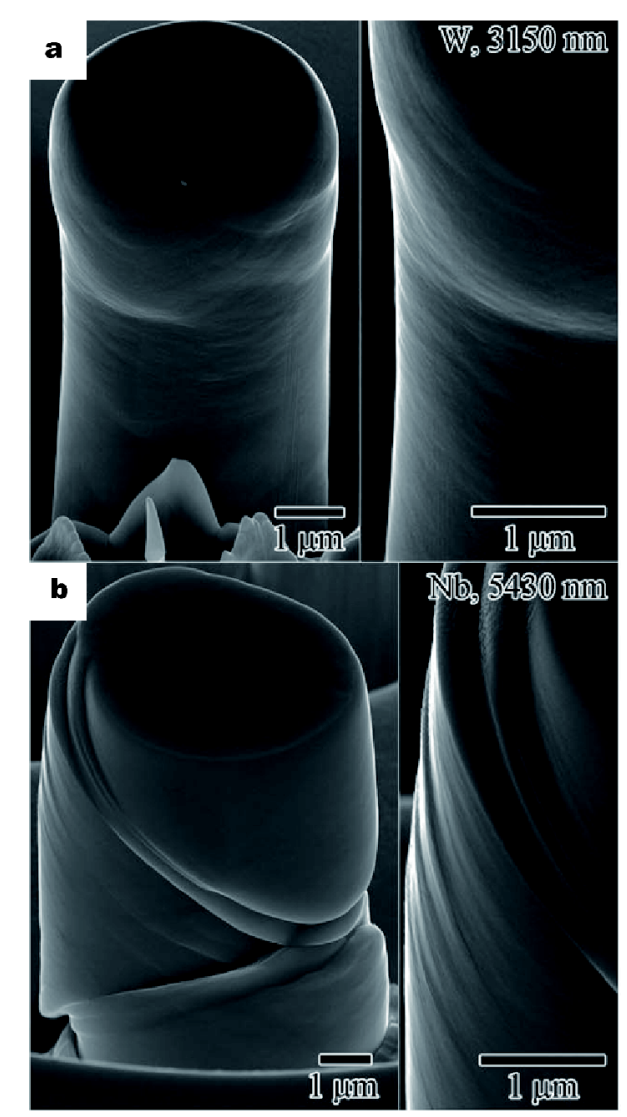

Figure 12 SEM images of (a) W and (b) Nb single crystalline pillars. W presented wavy slip morphology, while $\mathrm{Nb}$ shows localized slip, reproduced from Ref. [28], Copyright 2009, American Physical Society.

example, in situ compression and tensile deformation of single crystalline $\mathrm{Nb}$ with diameters in a range of 200 to $900 \mathrm{~nm}$ show parallel slip lines on their side-wall surface (see Fig. 12b) [59], which indicates a preferential confinement of dislocations on a single slip system without cross-slip. It is proposed that the mean free path of screw dislocations is larger in small-sized $\mathrm{Nb}$ than that 
in its bulk counterpart, which favors localized plasticity over homogeneous plasticity. Additionally, the nonnegligible image force of small-sized metals can accelerate dislocation motion towards the free surface [59]. This phenomenon is quite prevailing in most BCC Mo, $\mathrm{V}, \mathrm{Nb}$ and $\mathrm{Ta}$ at small scales and is strikingly different from the plastic behavior of their bulk counterparts $[44,59,60,63,85,99]$. However, such plastic behavior transition is not obvious in $\mathrm{W}$, which may be due to its high $T_{c}$ (see Table 1). Researchers [99] compared different plastic morphologies of $\mathrm{W}$ pillars with decreasing diameters at a range of temperatures. It turns out that $\mathrm{W}$ presents a localized plastic deformation instead of wavy morphology only at a temperature higher than $T_{\mathrm{c}}$.

\section{Size-dependent strain rate sensitivity}

Strain rate sensitivity (SRS) is an important mechanical parameter that represents the sensitivity of flow stress to strain rate. It is described in the equation below:

$$
m=\frac{\sqrt{3} k T}{\sigma V^{*}},
$$

where $m$ is SRS exponent, which is used to describe the size dependence of SRS, $k$ is Boltzmann constant, $T$ is absolute temperature, $\sigma$ is applied uniaxial stress and $V^{*}$ is thermal activation volume [113].

FCC metals always present an inverse proportion of SRS to intrinsic size (grain size) and extrinsic size (sample dimension) $[44,55,114-116]$. In BCC metals, the SRS is in a more complicated situation: the flow stress of smallsized samples is insensitive to strain rate; for large samples, their flow stress is significantly influenced by strain rate. As mentioned in the section of "Sizedependent yield strength and flow stress", flow stress is affected by local energy barrier. The thermal activation of dislocations helps overcome the energy barrier and thereby reaches a small flow stress. Previous work [116] indicates that the mobility of screw dislocations in BCC metals is the rate controlling factor in plastic deformation. The double-kink mechanism of screw dislocations [117], by which the thermal activation can raise one segment of a straight screw dislocation to the next adjacent energy valley inducing two edge-type dislocation kinks at both sides of the screw segment, is considered to influence SRS significantly at low temperature (below $T_{\mathrm{c}}$ ). For BCC metals, the activation volume decreases with increasing stress. According to the Hall-Petch relationship, reducing sample size increases flow stress and yield strength, thereby corresponding to a smaller activation volume. When the sample size is below the critical length of the double kinks, i.e., the distance between two edge segments, which is usually $\sim 1 \mu \mathrm{m}$ [118], the decrease of the activation volume with increasing stress could be negligible and the activation volume tends to be a constant. According to Equation (9), due to the rapid increase of stress and slow decrease of activation volume, the SRS exponent $m$ thus decreases with decreasing sample size. It has been reported that the value of $m$ for a-Fe drops dramatically from $\sim 0.06$ to $\sim 0.005$ when sample size is reduced from $1 \mu \mathrm{m}$ to $200 \mathrm{~nm}$ [108]. With further decrease of sample size to smaller than $50 \mathrm{~nm}$ [113], dislocation type is no longer a controlling factor to SRS [83,100], and the mobility of screw and edge dislocations under high stress are about the same as suggested by MD simulations for BCC Mo [100]. Under these circumstances, a further consideration of stress contribution is necessary. It has been predicted that, with further decreasing size, dislocation nucleation from free surface or creep, both having very small activation volumes, will be the major contribution for the high strength of samples. Therefore, $m$ will ideally approach to 1 and superplasticity will occur $[108,119]$.

\section{Size-dependent defect-mediated plastic mechanisms}

Plastic deformation of metallic materials is usually accommodated by dislocation slip and/or deformation twinning. For most single crystalline and polycrystalline FCC and BCC metals, there are four factors affecting the propensity of deformation twinning: (1) stacking fault energy, (2) strain rate, (3) deformation temperature, and (4) grain size (or sample size for single crystals). For BCC metals, full dislocation-mediated plasticity overwhelms twinning-mediated plasticity in most situations due to their large stacking fault energy. For example, the stacking fault energy of BCC Mo is calculated as more than $1 \mathrm{~J} \mathrm{~m}^{-2}$ [89], which is one order of magnitude higher than that of FCC $\mathrm{Al}\left(0.14 \mathrm{~J} \mathrm{~m}^{-2}\right)$ [120]. With such a very high stacking fault energy in BCC metals, deformation twinning can only occur under high strain rate or low deformation temperature [4], which is very different from that of FCC metals [121]. Therefore, most of simulations [67-72] and experiments [44,58-60,63,85,98,100-103] pay more attention to full dislocation-mediated plasticity than twinning-mediated plasticity in BCC metals. Recently, orientation dependence and size dependence of twinning-mediated plasticity in single crystalline metals have been confirmed by simulation work $[89,122,123]$, in which deformation twinning can occur in small-sized BCC metallic nanowires (usually with a diameter of several nanometers) at certain loading directions. However, simulations are usually for high 


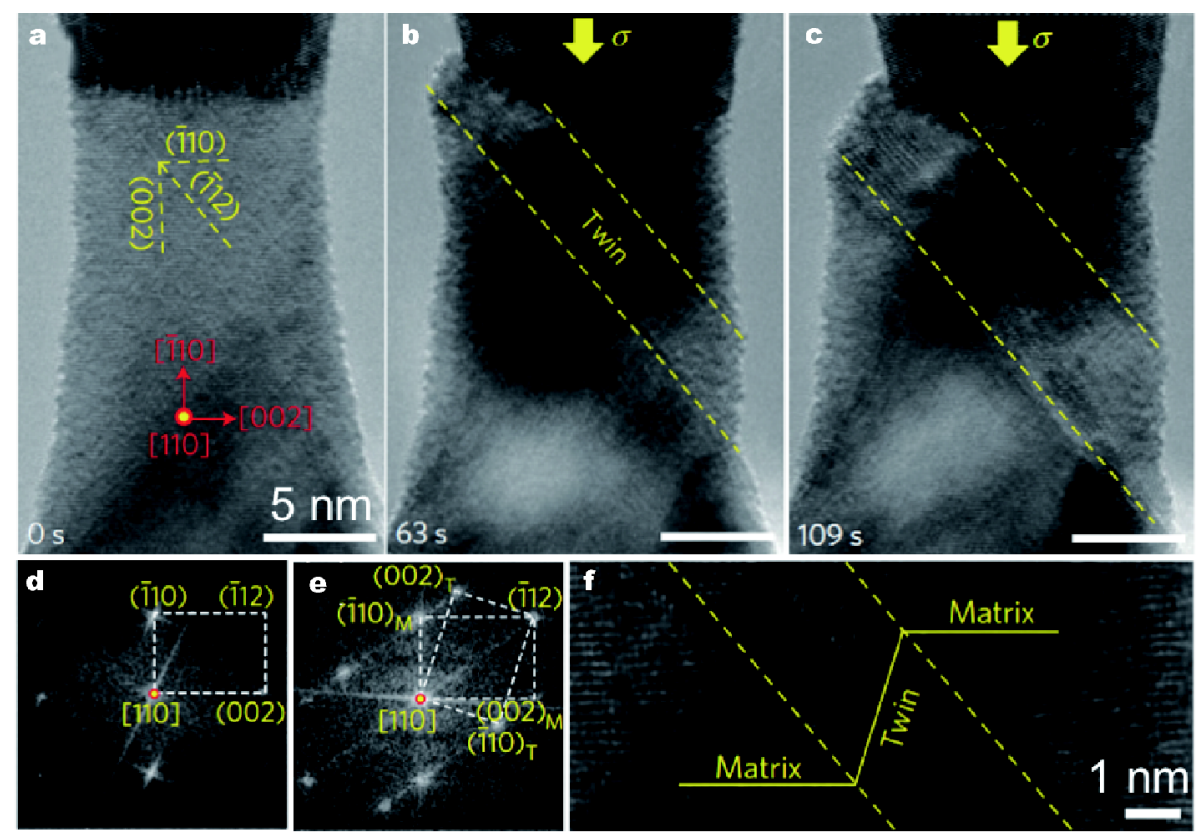

Figure 13 Deformation twinning in a W bicrystal nanowire under compression along the [1110] direction. (a-c) Sequential TEM images showing deformation twinning. (d, e) Fast Fourier transformation patterns of the original W bicrystal and the deformation twin, respectively. (f) Magnified TEM image showing the deformation twin, reproduced from Ref. [124], Copyright 2015, Nature Publishing Group.

strain rates and their results may not be applicable to the low strain-rate situations. Therefore, experimental evidence of deformation twinning in BCC metals is critical. Recent in situ straining high resolution TEM (HRTEM) investigation of BCC $\mathrm{W}$ nanowires with diameters of $\sim 15 \mathrm{~nm}$ [124] showed that, different from the full dislocation-mediated plasticity in large BCC samples, twin embryo nucleated from the intersection between grain boundary and free surface, and deformation twinning was a dominant plastic path under certain loading directions at room temperature and low strain rates, as shown in Fig. 13.

Molecular dynamics (MD) simulations for a large range of loading stresses showed that the behavior of dislocations in BCC Fe depends strongly on the stress level [125]. Under low stress $(<600 \mathrm{MPa})$, full dislocations move smoothly through the formation and migration of atomic-sized kinks. Under medium stress between $600 \mathrm{MPa}$ and $1.8 \mathrm{GPa}$, full dislocations become rugged and move in a more jerky way. Under high stress $(>1.8 \mathrm{GPa})$, full dislocations tend to dissociate into partial dislocations to form deformation twins. A full screw dislocation with Burgers vector of $1 / 2[111]$ prefers to dissociate into three partial dislocations with Burgers vector of $1 / 6[111]$ on three consecutive (112) planes under a large external stress of larger than $1.8 \mathrm{GPa}$, forming a twin embryo with a thickness of three atomic layers. Regarding the prominent size effect on flow stress of BCC metals, the transition from full dislocationmediated plasticity to twinning-mediated plasticity is thus reasonable when decreasing the sample diameter from hundreds of nanometers to $15 \mathrm{~nm}$. The compressive flow stress in previous experiments [124] is much larger than 1.8 GPa, indicating a preferable stress state for twinningmediated plasticity.

Nevertheless, the size effect on the plastic path does not apply to all situations [109-112,124]. MD simulations [124] confirmed a strong link between shear stress and the competition of the full dislocation slip and deformation twinning. Therefore, Schmid factor plays a significant role in the selection of the plastic path, leading to a loading orientation dependence of the plastic deformation mechanism. If the resolved shear stress on a twinning system is comparable to or smaller than that on dislocation slip systems, the lateral expansion of twin embryo will be impeded by the emission of full dislocations, consequently leading to a full dislocationmediated plasticity. It should be noted that the impact of resolved shear stress on the selection of plastic path can make sense only when a resolve shear stress is larger than the CRSS of the corresponding slip systems. If the resolved shear stress does not overcome the CRSS, no competition of plastic deformation mechanisms will occur. 

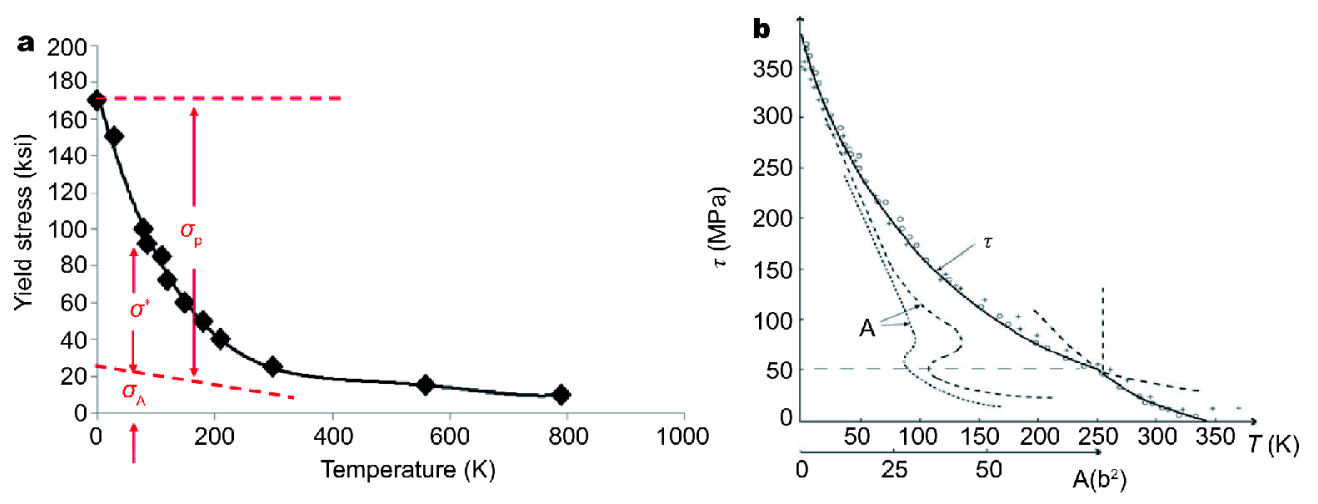

Figure 14 Temperature dependence of yield stress in (a) Ta, reproduced from Ref. [84], Copyright 1977, Elsvier and (b) $\alpha$-Fe, reproduced from Ref. [74], Copyright 2010, Elsevier.

\section{THE TEMPERATURE DEPENDENCE OF THE SIZE EFFECT ON SINGLE CRYSTALLINE BCC METALS}

Stress responses of BCC metals are significantly affected by temperature, which arises from the temperature dependence of the mobility of screw dislocations. Screw dislocations in BCC metals encounter large Peierls stress (the lattice resistance for dislocations at $0 \mathrm{~K}$ [84]) below the critical temperature due to their unusual non-planar core structure. Peierls stress can be overcome by thermal activation. Therefore, stress response in BCC metals can be divided into two parts: thermal stress and athermal stress $[84,126,127]$. Thermal stress is the stress to overcome Peierls stress for dislocation motion. Athermal stress results from dislocation nucleation and work hardening. Temperature rise can help overcome Peierls stress and this reduces the thermal stress. However, such temperature-dependent stress response is limited. When temperature reaches the critical temperature, thermal stress is negligible. Athermal stress, which is insensitive to temperature, thus dominates the total stress responses.

A further illustration of the temperature dependence of yield strength is presented in Fig. 14. Fig. 14a shows that the thermal stress $\sigma^{*}$ of BCC Ta decreases with increasing temperature, while athermal stress $\sigma_{\mathrm{A}}$ is insensitive to variation of temperature. Thermal stress accounts for significant contribution to total stress below the critical temperature of $450 \mathrm{~K}$, after which thermal stress $\sigma^{*}$ no longer dominates the measured stress and the total stress is nearly a constant that is equal to the athermal stress. Additionally, $\sigma_{\mathrm{P}}$ is Peierls stress. Fig. $14 \mathrm{~b}$ presents a similar correlation between thermal stress $\tau$ and temperature in $\alpha$-Fe. Thermal stress decreases to 0 at $340 \mathrm{~K}$, which is the critical temperature of $\alpha-\mathrm{Fe}$ (see Table 1). Such decreasing response of thermal stress to temperature

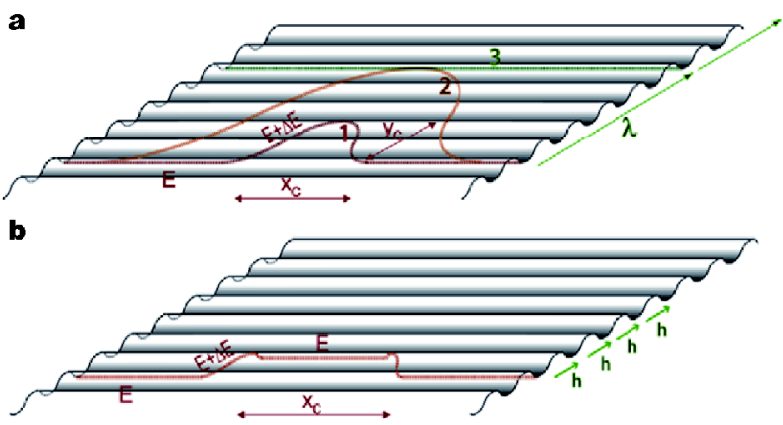

Figure 15 Schematics of (a) jerky motion and (b) kink-pair motion of screw dislocation motion, reproduced from Ref. [74], Copyright 2010, Elsevier.

is attributed to the increased activation volume. As indicated in Fig. 14b, activation area $A$, which is positively scaled with activation volume $V^{*}$, increases with temperature, implying that activation area directly affects thermal stress. A sudden change of slope of stress $-A$ curve at $250 \mathrm{~K}$ corresponds to a minimum dependence of the thermal stress on activation area, which means a change of dislocation mechanism.

\section{The temperature effect on the motion of screw dislocations} The behavior of screw dislocations in BCC metals is temperature-dependent and this influences significantly their stress responses. At the low temperature regime below $T_{\mathrm{c}}$, screw dislocations always behave in two forms to sustain plastic deformation: jerky motion [74] and kink-pair motion $[117,118,123]$, both require thermal activation during plastic deformation. The local minimum dependence of the thermal stress on the activation area in Fig. 14b represents a transition from jerky motion to kink-pair motion. Fig. 15 shows the difference in these two motion types. Here $E$ is the energy of Peierls valley; $\Delta E$ is the difference of core energy between mobile 

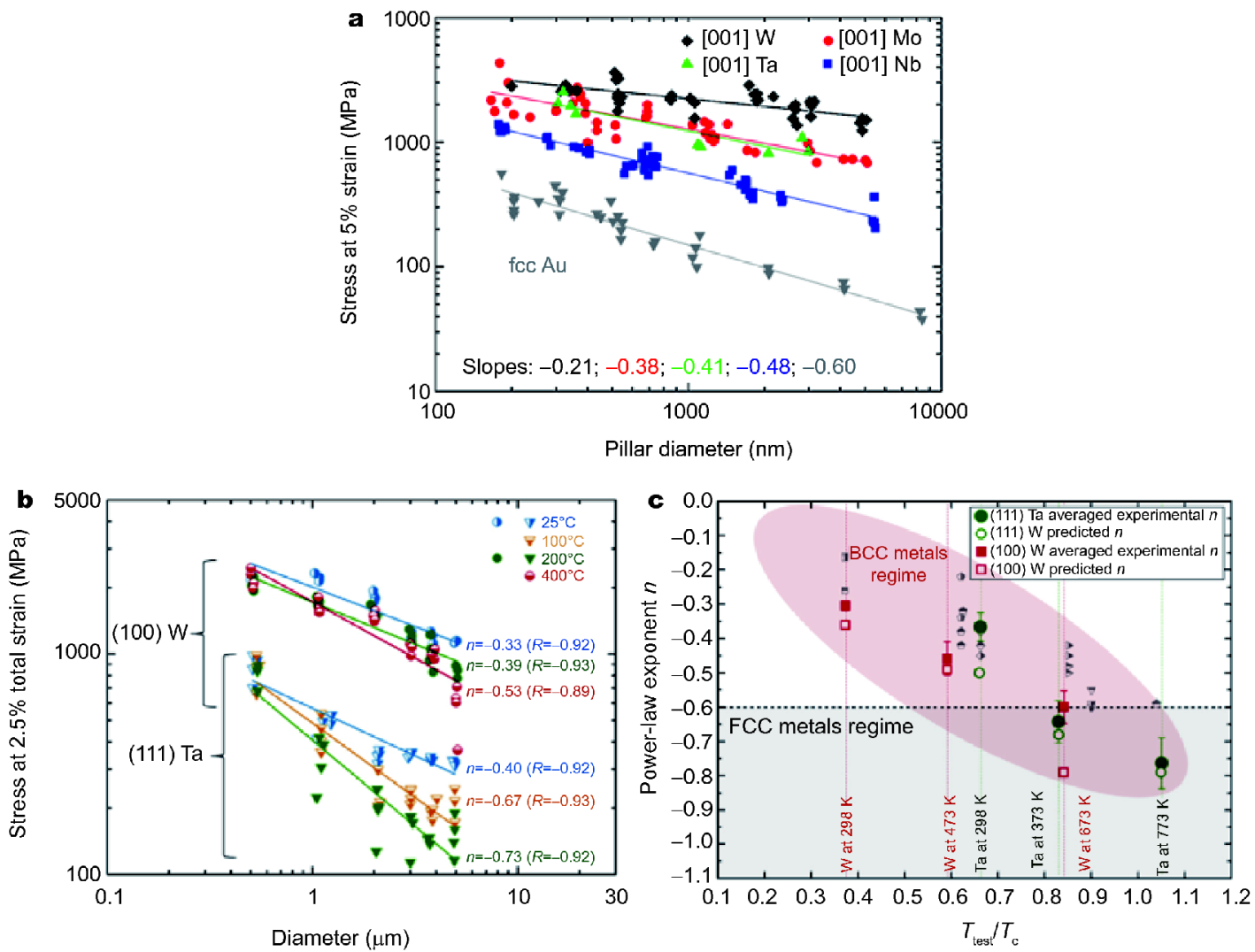

Figure 16 (a) Comparison of flow stress at 5\% strain among BCC metals and FCC Au at room temperature, reproduced from Ref. [28], Copyright 2009, American Physical Society. (b) Size effect on flow stress of W and Ta at different temperatures, reproduced from Ref. [98], Copyright 2014, Springer Nature. (c) Power-law exponent $n$ as a function of $T_{\text {test }} / T_{\mathcal{c}}$, reproduced from Ref. [99], Copyright 2009, American Physical Society.

dislocations and sessile dislocations; $x_{c}$ is the bulge width; $y_{\mathrm{c}}$ and $h$ are height of bulge and kink-pair, indicating two different threshold configurations; $\lambda$ is the actual jump length that is used to compare to $y_{\mathrm{c}}$ and $h$ for judging which motion is preferred. For jerky motion in $\alpha$-Fe (Fig. 15a) [74], a part of a screw dislocation can glide freely over Peierls valleys below $250 \mathrm{~K}$. For kink-pair motion (Fig. 15b), a small segment of a screw dislocation is thermally activated from one Peierls valley to the neighboring valley, creating two edge kinks on both sides of screw segment. The edge kinks can glide on the opposing directions and finally sink on free surface or annihilate via dislocation interaction. The threshold configurations of jerky motion and kink-pair motion in $\alpha$-Fe change with temperature, indicating a competition between jerky motion and kink-pair motion. When $T>T_{\mathfrak{c}}$, Peierls stress for screw dislocations is negligible, implying motion with equal velocity of screw dislocations and edge dislocations in BCC metals.

The temperature-dependent mobility of screw disloca- tions can significantly affect the stress responses in BCC metals. When the mobility of screw dislocations is much lower than edge dislocations (at a temperature $<T_{\mathrm{c}}$ ), thermal stress dominates the total stress. The overall flow stresses during the plastic deformation of BCC metals at this temperature can be much higher than that in FCC metals. When the mobility of screw dislocations is as pronounced as that of edge dislocations, the stress response of BCC metals is similar to that of FCC metals.

The temperature effect on size strengthening exponent $n$ Although the values of $n$ are negative in some references $[28,60,85,99,108]$, only the absolute values are considered in this review. As mentioned in the section "Sizedependent yield strength and flow stress", the power-law exponent $n$ in Equation (6) for FCC metals is in an order of 0.5 to 1 , which indicates significant size dependence of stress response. In contrast, BCC metals always show a relatively weak size effect on their flow stress. This phenomenon has been attributed to the strong tempera- 
ture-dependent mobility of screw dislocations and the intrinsic critical temperature in BCC metals [28,44, $85,99,107,108]$. The size strengthening exponent $|n|$ decreases with increasing critical temperature as shown Table 1 and Fig. 16a [28], in which the slopes of curves represent corresponding exponents $n$. Obviously, $\mathrm{Nb}, \mathrm{V}$ and $\alpha-F e$, which have low critical temperatures below $380 \mathrm{~K}$, have strong size effect on their flow stress responses. The absolute values of exponent $n$ of these BCC metals are in the range of 0.48 to $0.93[28,85,107$, 108], which is close to that of FCC Au (0.6). On the contrary, Ta, Mo and $\mathrm{W}$, which have high critical temperatures, show a weak size dependence of flow stresses, with exponents $n$ in the order of 0.21 to 0.44 [28, 85]. Such difference among BCC metals arises from the strong impact of Peierls stress on the motion of screw dislocations. Screw dislocations in BCC metals with high critical temperatures encounter large lattice resistance due to small activation volume at the ambient temperature $(300 \mathrm{~K})$. The thermal stress accounts for a dominant fraction in total stress responses if screw dislocations are thermally-activated. Large thermal stress has weakened the size-dependent strengthening arising from the weakest-link and dislocation starvation mechanisms. For BCC metals with low critical temperatures $(\mathrm{Nb}, \mathrm{V}, \mathrm{a}-\mathrm{Fe})$, the activation volume is large at room temperature. Therefore, stress responses of those BCC metals are insensitive to dislocation types. In this situation, BCC metals have prominent size effects on their stress responses as pronounced as FCC metals during plastic deformation [44].

Another interesting point is that the difference of the flow stresses among various BCC metals, when the sample size is small enough, is negligible (as shown in Fig. $16 \mathrm{~b}, \mathrm{c})$. That is because large surface to volume ratio can facilitate dislocation escape from free surface and thus requires similar stress for dislocation nucleation from surface among different metals. It also seems that the temperature dependence of flow stress for each BCC metal is negligible when the sample size decreases to the nanoscale, meaning that the mobility of screw dislocations is insensitive to temperature at this size regime. Because a large surface to volume ratio in a small sample can enhance the mobility of screw dislocations by kink nucleation on surface and thereby reduce the temperature impact on stress responses [28].

\section{The temperature effect on size-dependent SRS}

The temperature effect on the size-dependent SRS can also be considered using the activation volume. The trends that activation volume decreases significantly with stress in large-scaled samples and keeps nearly a constant when the sample size decreases to the submicron scale has been discussed in the section of "Size-dependent strain rate sensitivity". Since the SRS exponent $m$ is defined as $\mathrm{d} \ln \tau / \mathrm{d} \ln \varepsilon$ [113], it is thus easy to extrapolate the correlation between activation volume $V^{*}$ and applied uniaxial stress $\sigma$ from Equation (9). To reveal the stress dependence of activation volume, a further derivation is made as shown in Equation (10):

$$
\frac{\mathrm{d} V^{*}}{\mathrm{~d} \sigma}=\frac{-3 k T \ln \varepsilon}{(\sigma-C)^{2}},
$$

where $C$ is a constant, $\varepsilon$ is strain rate, other parameters are the same as those in Equation (9). Assuming that a BCC sample with a certain size is loaded at a constant strain rate, increasing temperature can increase the dependence of activation volume $V^{*}$ on stress (an increase in $\left.-3 k T \ln \varepsilon /(\sigma-C)^{2}\right)$. This means that $V^{*}$ decreases more sharply with increasing temperature at high temperature than at low temperature, leading to more pronounced dependence of SRS exponent $m$ on sample size.

\section{The temperature effect on size-dependent plastic morphology}

It is mentioned that there is a transition of plastic morphology from wavy slip to localized slip with decreasing the sample size of BCC metals in the section of "Size-dependent plastic deformation morphology". Such transition is also dependent on temperature $[98,99]$. Cross-slip of screw dislocations, the main reason leading to wavy morphology, can be limited at high temperatures near or above $T_{c}$. The motion of screw dislocations is much easier at temperatures above $T_{\mathrm{c}}$ than at low temperatures, so that highly thermally activated screw dislocations can slide on its original slip plane without cross-slip and this leads to localized slip in BCC metals. Therefore, localized slip occurs even at large scale at high temperatures (see the section of "Size-dependent plastic deformation morphology"). A typical example is presented by Ref. [99], in which a W pillar $\left(T_{c}=800 \mathrm{~K}\right)$ with a diameter of $5 \mu \mathrm{m}$ only exhibits localized deformation at temperatures above $673 \mathrm{~K}$. Although the test temperature was below $T_{c}$, the cross-slip of screw dislocations was impeded by both thermal activation and free surface assistance. Consequently, localized slip is favored during plastic deformation in BCC metals in a large range of sample size at high temperature. The morphology transition size can be large at high temperatures. 


\section{SIZE EFFECT ON THE PLASTICITY OF POLYCRYSTALLINE BCC METALS}

In polycrystalline BCC metals, grain size influences the plasticity significantly. As mentioned in the section of "GB-mediated plasticity in BCC metals", GB-mediated plastic mechanisms in BCC metals are similar with those in FCC materials. Hall-Petch relationships exist in both BCC and FCC polycrystalline materials at certain ranges of grain sizes. As grain size decreases to a small scale), the "soften" phenomenon occurs due to collective GB activities in lieu of intra-grain dislocation activities. Additionally, SRS of BCC polycrystalline metals scales with grain size $[116,128,129]$. This distinguishing phenomenon is also related to defects in BCC grains. In the following subsections, the grain size-effect on flow stress and SRS of BCC polycrystalline metals will be reviewed in detail.

\section{Size effect on flow stress of polycrystalline bcc metals}

For BCC metals, the strongest size also exists, below which the Hall-Petch relationship is no longer applicable to describe the grain size effect on strength. Fig. 17 concludes the size effect on the flow stress of polycrystalline $\mathrm{W}, \mathrm{Ta}$ and $\alpha-\mathrm{Fe}$, including both nanocrystals and microcrystals, at $6 \%$ strain during compression and tensile tests [91,116,128,130-137]. A trend of increasing flow stress is obvious with grain size decreasing to, for instance, $10 \mathrm{~nm}$ for $\mathrm{Ta}$ and $\alpha-\mathrm{Fe}$ in tension and $100 \mathrm{~nm}$ for Ta in compression. When grain size is larger than the threshold value, dislocations are emitted from GBs and pile up in front of the other side of the GBs. When grain size is smaller the threshold value, GB-mediated plasticity, including GB sliding and rotation, occurs instead of intra-grain dislocation activities due to the increased volume fraction of GBs, leading to a "softening" phenomenon, which is called an inverse Hall-Petch relationship. This phenomenon also applies to materials of other crystalline systems including FCC [44,138-140].

In addition, the strongest size also indicates the most brittle size $[141,142]$. The ductility of BCC nanocrystalline materials firstly decreases with decreasing grain size (but still larger than the strongest grain size). It is because that at this size range, GBs are obstacles for dislocation motion and also sink for dislocations. Increasing grain size results in easier plastic deformation and also increases the capability of dislocation storage. The latter increases strain hardening rate and therefore ductility. However, when the grain size is small enough for GBmediated plasticity (smaller than the strongest grain size), GB activities can accommodate large strain with grain

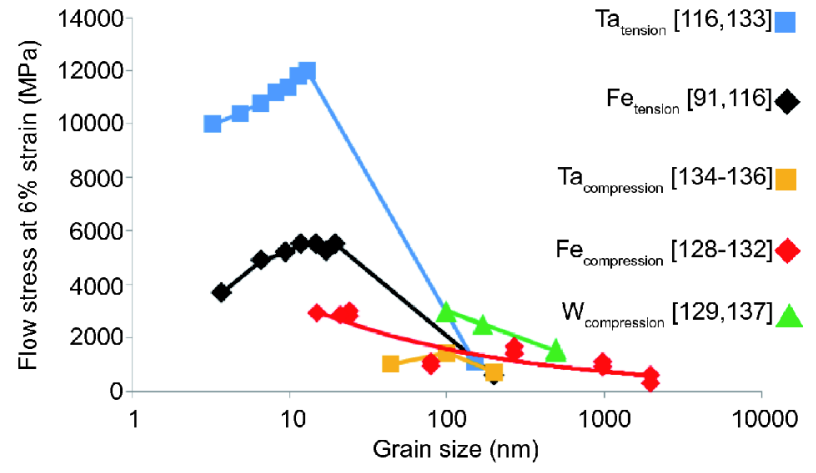

Figure 17 Flow stress of polycrystalline $\mathrm{Ta}, \mathrm{a}-\mathrm{Fe}$ and $\mathrm{W}$ at $6 \%$ strain during compression and tension tests.

size decreasing and thereby increase the resistance to crack propagation. For those with grain sizes near the strongest size, cracks nucleate easily at GBs under external stress due to coalescence of small voids. MD simulations of nanocrystalline Mo suggested that the interaction between twin boundaries (TBs) and GBs can also lead to crack nucleation [143,144]. Deformation twinning is an important plastic mechanism in nanocrystalline BCC metals due to the triaxial stress at GBs [143]. When a deformation twin in one grain reaches a $\mathrm{GB}$, it is stopped by the GB that leads to stress concentration and an intergranular crack can nucleate at the GB.

\section{Size effect on the SRS of polycrystalline BCC metals}

The size effect on the SRS of single crystalline BCC metals has been discussed before. Theoretical analyses were introduced to explain the inverse proportion of the exponent $m$ of SRS to sample size. Basically, the activation volume is considered as the fundamental factor to influence SRS. For polycrystalline BCC metals, positive correlation between $m$ and grain size also exists $[116,128,129]$. Direct correlation between this size effect and dislocation type in BCC Mo grains was revealed. The relationship between size-dependent dislocation type and the corresponding density is shown in Fig. 18 [145]. While screw-type dislocations control the plastic deformation of large-sized BCC metals, edge- and mixed-type dislocations governed the plasticity of nanocrystalline BCC metals when grain sizes decrease to a certain value, which is $150 \mathrm{~nm}$ in Fig. 18. When grain sizes are smaller than $30 \mathrm{~nm}$, most grains are in dislocation-free state due to dislocation annihilation at GBs. For grain sizes larger than $30 \mathrm{~nm}$, the relationship between the size-dependent SRS exponent $m$ and dislocation type can be described as below: 


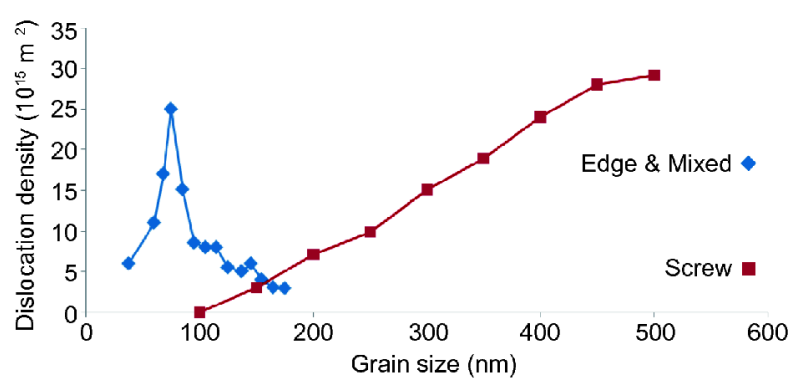

Figure 18 Variation of dislocation densities with grain size for screw dislocations, edge dislocations and mixed dislocations, reproduced from Ref. [145], Copyright 2013, Taylor \& Francis Group.

$$
\frac{1}{m}=\frac{\partial \ln \left(\rho_{\mathrm{s}} v_{\mathrm{s}}+\rho_{\mathrm{e}} v_{\mathrm{e}}\right)}{\partial \ln \tau}
$$

where $m$ is SRS exponent, $\rho_{\mathrm{s}}$ is the density of screw dislocations, $\rho_{\mathrm{e}}$ is the density of the edge component of non-screw dislocations, $v_{s}$ is the speed of screw dislocations, $v_{\mathrm{e}}$ is the speed of the edge component of other dislocations, $\tau$ is shear stress. For coarse-grained polycrystalline BCC metals, the density of screw dislocations overwhelms the density of edge dislocations, $\rho_{\mathrm{s}} v_{\mathrm{s}}>>\rho_{\mathrm{e}} v_{\mathrm{e}}$. Therefore, screw dislocation controls material's plasticity, leading to large $m$ (details have been discussed in the section of "Size-dependent strain rate sensitivity". For nanocrystalline BCC metals, the density of edge and mixed dislocations is much larger than the density of screw dislocations. As mentioned in the section of "Sizedependent strain rate sensitivity", when screw dislocations are no longer the dominant factor to influence SRS of BCC metals, the activation volume is usually large, corresponding to small exponent $m$. Therefore, in small grains where edge and mixed dislocations govern the plasticity, the activation volume is larger than that in large grains, leading to smaller SRS exponent $m$. Further reducing grain sizes to below the strongest size, GB activities control the plastic deformation of polycrystalline BCC metals. According to previous work [134,145], $m$ is inversely proportional to grain size due to the GB activities. However, there is no applicable theoretical model to explain this phenomenon. As it has been revealed that $\mathrm{GB}$ activities are actually the results of $\mathrm{GB}$ dislocation motion [25], the variation of activation volumes of $\mathrm{GB}$ dislocations may be the direct reason for this unusual size effect. This issue is waiting for further investigation.

\section{CONCLUSIONS AND OUTLOOK}

The complicated size-dependent plasticity of small-sized single crystalline and polycrystalline BCC metals has been reviewed. The following conclusions are reached:

The yield strength and flow stress increase with the reduction of both sample size and grain size. In single crystalline BCC metals, two size-dependent strengthening mechanisms, the weakest-link mechanism and the dislocation starvation mechanism, were proposed to explain the size effect at different size ranges. The weakest-link mechanism, which applied to large-sized BCC metals, reveals that the length of dislocation sources influences significantly the stress response. The dislocation starvation mechanism is the dominant strengthening mechanism at small sizes. The sample size for the crossover of the two mechanisms depends on factors including dislocation density and applied stress. When the sample size decreases to a critical value, the size effect on yield strength and flow stress is mainly affected by free surface. In polycrystalline BCC metals, there exists a critical grain size where materials get their highest strength and poorest ductility. Below this critical value, an inverse Hall-Petch effect applies. Such size effects arise from the transition of plastic deformation mechanism from dislocationmediated to GB-mediated plasticity because decreasing grain size increases the volume fraction of GBs that facilitates GB activities.

The SRS and therefore its exponent $m$ increase with sample size in single crystals. This size effect is caused by the variation of the activation volume. For large BCC samples, screw dislocations are the controlling factor for the exponent $m$; for small samples, dislocation type is no longer a controlling factor because the activation volumes of edge dislocations and screw dislocations are equal. Theoretical investigations predict a reverse correlation between $m$ and sample size when the sample size is below $\sim 100 \mathrm{~nm}$. The size effect on SRS of polycrystalline BCC metals presents a similar trend. While the average grain size is below the critical value, the SRS exponent $m$ presents inverse proportion to grain size due to GB activities.

The plastic morphology and SHR depend significantly on sample size. Decreasing sample size to the micro- or submicron-scales leads to a morphologic transition from homogeneous to localized deformation. SHR is inversely scaled to sample size, which is attributed to the weakestlink mechanism. Both of these two size effects depend on $T_{c}$. For BCC metals with high $T_{c}$, the Peierls stress for screw dislocations is large at the ambient temperature, indicating more frequent cross-slip. The frequent crossslip contributes to a more wavy way on morphology and strengthens the size effect on SHR.

The plastic deformation mechanism undergoes a 
transition from dislocation-mediated to twinningmediated plasticity with decreasing single crystalline sample size. The transition is affected significantly by the relationship between the crystallographic orientation and stress direction that determines the resolved shear stresses. While deformation twinning in large-sized BCC metals usually occurs at low temperatures and/or high strain rates, twinning in nano-sized single crystalline BCC metallic pillars with diameters of $\sim 15 \mathrm{~nm}$ can occur at the ambient temperature and low strain rates.

The above size effects are affected by the experimental temperature and $T_{c}$. The behavior of BCC metals with low $T_{\mathrm{c}}$ is usually similar to FCC metals, presenting pronounced size effect on yield/flow stresses. The size effect of BCC metals with high $T_{c}$ is not obvious. Additionally, increasing temperature can contribute to more distinct dependence of SRS on sample size and also more preferred localized plastic deformation.

Although extensive efforts have been made to investigate the size effects on the plasticity and deformation mechanisms of BCC metals, which are a most important type of structural materials, their plastic deformation mechanisms are less understood compared to FCC materials. Many outstanding issues remain to be resolved. For single crystalline BCC materials, the critical size for the transition from screw dislocation slip to edge dislocation slip or vice versus, from dislocation slip to deformation twinning, and how deformation conditions such as temperature and strain rate affect the critical size remain unclear. Experimental evidence at the atomic resolution is needed to verify MD simulation results on the nucleation and migration of defects in nano-sized BCC metals. For polycrystalline BCC metals, the upperbound limit of strength and the crossover from dislocation-mediated plasticity to GB-mediated plasticity with reducing grain size has been studied mainly by computational simulations and stress responses [128,130-137]. Experimental evidence at the atomic resolution on the transition from Hall-Petch to inverse Hall-Petch relationship and the transition of plastic deformation mechanisms in nanocrystalline BCC metals is far from enough.

With the recent development of aberration-corrected TEM, an ultrahigh-resolution of sub-angstrom has been achieved, which provides a powerful tool for studying the structures of materials and the dynamic of structural evolutions. To gain thorough understanding of the deformation mechanisms and mechanical properties of small-sized BCC metals at different length scales, it is critical to significantly enhance our experimental capabilities in the following two aspects: (1) the capability in producing nanocrystalline materials with grain sizes down to a few nanometers and single crystalline nanowire/pillar samples with diameters also down to nanometers and with desirable defect structures and crystallographic orientations, and (2) in situ straining microscopy techniques. We need to develop techniques that enable both atomic scale imaging of samples in TEM and precise force-displacement measurement even for materials with diameters down to a few nanometers. The signal-to-noise ratios of currently available in situ straining TEM facilities are too low to acquire meaningful stress-strain data for nanowires/nanopillars with diameters of smaller than $10 \mathrm{~nm}$.

Finally, many materials for high temperature applications are of BCC structures. It is critical to understand the roles of BCC structures in impeding the nucleation and motion of dislocations and other defects that stabilizes the microstructures at high temperatures. Developing microscopy techniques to decode these mechanisms is very important for the design and development of new materials with excellent high-temperature mechanical performance and with low weight and low manufacturing cost.

Received 9 May 2018; accepted 20 June 2018; published online 10 August 2018

1 Baughman RH, Zakhidov AA, de Heer WA. Carbon nanotubesthe route toward applications. Science, 2002, 297: 787-792

2 Craighead HG. Nanoelectromechanical systems. Science, 2000, 290: 1532-1535

3 Zhu T, Li J. Ultra-strength materials. Prog Mater Sci, 2010, 55: 710-757

4 Zhu YT, Liao XZ, Wu XL. Deformation twinning in nanocrystalline materials. Prog Mater Sci, 2012, 57: 1-62

5 Wei B, Zheng K, Ji Y, et al. Size-dependent bandgap modulation of $\mathrm{ZnO}$ nanowires by tensile strain. Nano Lett, 2012, 12: 45954599

6 Zheng K, Han X, Wang L, et al. Atomic mechanisms governing the elastic limit and the incipient plasticity of bending Si nanowires. Nano Lett, 2009, 9: 2471-2476

7 Zheng K, Wang C, Cheng YQ, et al. Electron-beam-assisted superplastic shaping of nanoscale amorphous silica. Nat Commun, 2010, 1: 24

8 Zhang Y, Han X, Zheng K, et al. Direct observation of superplasticity of beta-SiC nanowires at low temperature. Adv Funct Mater, 2007, 17: 3435-3440

9 Han XD, Zhang YF, Zheng K, et al. Low-temperature in situ large strain plasticity of ceramic $\mathrm{SiC}$ nanowires and its atomic-scale mechanism. Nano Lett, 2007, 7: 452-457

10 Tian L, Cheng YQ, Shan ZW, et al. Approaching the ideal elastic limit of metallic glasses. Nat Commun, 2012, 3: 609

11 Wang J, Sansoz F, Deng C, et al. Strong Hall-Petch type behavior in the elastic strain limit of nanotwinned gold nanowires. Nano Lett, 2015, 15: 3865-3870

12 Sylwestrowicz W, Hall EO. The deformation and ageing of mild 
steel. Proc Phys Soc B, 1951, 64: 495-502

13 Petch NJ. The cleavage strength of polycrystals. J Iron Steel Inst, 1953, 174: 25-28

14 Yue Y, Liu P, Zhang Z, et al. Approaching the theoretical elastic strain limit in copper nanowires. Nano Lett, 2011, 11: 3151-3155

15 Wang L, Liu P, Guan P, et al. In situ atomic-scale observation of continuous and reversible lattice deformation beyond the elastic limit. Nat Commun, 2013, 4: 2413

16 Deng Q, Cheng Y, Yue Y, et al. Uniform tensile elongation in framed submicron metallic glass specimen in the limit of suppressed shear banding. Acta Mater, 2011, 59: 6511-6518

17 Cheng GM, Xu WZ, Jian WW, et al. Dislocations with edge components in nanocrystalline bcc Mo. J Mater Res, 2013, 28 : 1820-1826

18 Wang L, Han X, Liu P, et al. In situ observation of dislocation behavior in nanometer grains. Phys Rev Lett, 2010, 105: 135501

19 Argon AS, Yip S. The strongest size. Philos Mag Lett, 2006, 86: 713-720

20 Wang YB, Wang LF, Joyce HJ, et al. Super deformability and Young's modulus of GaAs nanowires. Adv Mater, 2011, 23: 13561360

21 Chen Y, Gao Q, Wang Y, et al. Determination of Young's modulus of ultrathin nanomaterials. Nano Lett, 2015, 15: 52795283

22 Cao R, Deng C. The ultra-small strongest grain size in nanocrystalline Ni nanowires. Scripta Mater, 2015, 94: 9-12

23 Schiøtz J, Jacobsen KW. A maximum in the strength of nanocrystalline copper. Science, 2003, 301: 1357-1359

24 Schiøtz J, Di Tolla FD, Jacobsen KW. Softening of nanocrystalline metals at very small grain sizes. Nature, 1998, 391: 561-563

25 Wang L, Teng J, Liu P, et al. Grain rotation mediated by grain boundary dislocations in nanocrystalline platinum. Nat Commun, 2014, 5: 4402

26 Wang L, Zhang Z, Ma E, et al. Transmission electron microscopy observations of dislocation annihilation and storage in nanograins. Appl Phys Lett, 2011, 98: 051905

27 Yu Q, Shan ZW, Li J, et al. Strong crystal size effect on deformation twinning. Nature, 2010, 463: 335-338

28 Schneider AS, Kaufmann D, Clark BG, et al. Correlation between critical temperature and strength of small-scale bcc pillars. Phys Rev Lett, 2009, 103: 105501

29 Dimiduk DM, Uchic MD, Parthasarathy TA. Size-affected singleslip behavior of pure nickel microcrystals. Acta Mater, 2005, 53: 4065-4077

$30 \mathrm{Wu} \mathrm{Z}$, Zhang YW, Jhon $\mathrm{MH}$, et al. Nanowire failure: long = brittle and short = ductile. Nano Lett, 2012, 12: 910-914

31 Lu Y, Xiang S, Xiao L, et al. Dislocation "bubble-like-effect" and the ambient temperature super-plastic elongation of bodycentred cubic single crystalline molybdenum. Sci Rep, 2016, 6: 22937

32 Yue Y, Chen N, Li X, et al. Crystalline liquid and rubber-like behavior in $\mathrm{Cu}$ nanowires. Nano Lett, 2013, 13: 3812-3816

33 Yue Y, Liu P, Deng Q, et al. Quantitative evidence of crossover toward partial dislocation mediated plasticity in copper single crystalline nanowires. Nano Lett, 2012, 12: 4045-4049

34 Shan ZW, Mishra RK, Syed Asif SA, et al. Mechanical annealing and source-limited deformation in submicrometre-diameter $\mathrm{Ni}$ crystals. Nat Mater, 2008, 7: 115-119

35 Kiener D, Minor AM. Source truncation and exhaustion: insights from quantitative in situ TEM tensile testing. Nano Lett, 2011, 11:
3816-3820

36 Wang L, Teng J, Sha X, et al. Plastic deformation through dislocation saturation in ultrasmall Pt nanocrystals and its in situ atomistic mechanisms. Nano Lett, 2017, 17: 4733-4739

37 Wang L, Kong D, Xin T, et al. Deformation mechanisms of bent Si nanowires governed by the sign and magnitude of strain. Appl Phys Lett, 2016, 108: 151903

38 Wang L, Zheng K, Zhang Z, et al. Direct atomic-scale imaging about the mechanisms of ultralarge bent straining in Si nanowires. Nano Lett, 2011, 11: 2382-2385

39 Han X, Zheng K, Zhang Y, et al. Low-temperature in situ largestrain plasticity of silicon nanowires. Adv Mater, 2007, 19: 21122118

40 Liu P, Mao SC, Wang LH, et al. Direct dynamic atomic mechanisms of strain-induced grain rotation in nanocrystalline, textured, columnar-structured thin gold films. Scripta Mater, 2011, 64: 343-346

41 Cao A, Wei Y, Ma E. Grain boundary effects on plastic deformation and fracture mechanisms in $\mathrm{Cu}$ nanowires: Molecular dynamics simulations. Phys Rev B, 2008, 77: 195429

42 Wang L, Zhang Z, Han X. In situ experimental mechanics of nanomaterials at the atomic scale. NPG Asia Mater, 2013, 5: e40

43 Han X, Wang L, Yue Y, et al. In situ atomic scale mechanical microscopy discovering the atomistic mechanisms of plasticity in nano-single crystals and grain rotation in polycrystalline metals. Ultramicroscopy, 2015, 151: 94-100

44 Greer JR, De Hosson JTM. Plasticity in small-sized metallic systems: Intrinsic versus extrinsic size effect. Prog Mater Sci, 2011, 56: 654-724

45 Li D, Shu X, Kong D, et al. Revealing the atomistic deformation mechanisms of face-centered cubic nanocrystalline metals with atomic-scale mechanical microscopy: A review. J Mater Sci Tech, 2018, 34: 2027-2034

46 Wieduwilt T, Tuniz A, Linzen S, et al. Ultrathin niobium nanofilms on fiber optical tapers-a new route towards low-loss hybrid plasmonic modes. Sci Rep, 2015, 5: 17060

47 Thieme K, Avramov I, Rüssel C. The mechanism of deceleration of nucleation and crystal growth by the small addition of transition metals to lithium disilicate glasses. Sci Rep, 2016, 6: 25451

48 Hao S, Cui L, Jiang D, et al. A transforming metal nanocomposite with large elastic strain, low modulus, and high strength. Science, 2013, 339: 1191-1194

49 Follansbee PS. Application to BCC Metals and Alloys. Berlin: John Wiley \& Sons, Inc., 2014

50 Acton QA. Advances in Nonaotechnology Research and Aplicaition. Scholary Editions, 2011

51 Acton QA. Heavy Metals: Advances in Research and Application. Scholary Editions, 2013

52 Callister WDJ. Fundamentals of Materials Science and Engineering. Berlin: John Wiley \& Sons, 2001, 34: 824

53 International A. https://compass.astm.org/Standards/HISTORICAL/E8E8M-13.htm

54 Compass A. https://compass.astm.org/EDIT/html_annot.cgi? D638+14

55 Schwaiger R, Moser B, Dao M, et al. Some critical experiments on the strain-rate sensitivity of nanocrystalline nickel. Acta Mater, 2003, 51: 5159-5172

56 BRUKER. https://www.bruker.com/products/surface-and-dimensional-analysis/nanomechanical-test-instruments.html

57 Nanomechanics. http://nanomechanicsinc.com/insem-systems- 
page/

58 Kim JY, Greer JR. Tensile and compressive behavior of gold and molybdenum single crystals at the nano-scale. Acta Mater, 2009, 57: 5245-5253

59 Kim JY, Jang D, Greer JR. Insight into the deformation behavior of niobium single crystals under uniaxial compression and tension at the nanoscale. Scripta Mater, 2009, 61: 300-303

60 Brinckmann S, Kim JY, Greer JR. Fundamental differences in mechanical behavior between two types of crystals at the nanoscale. Phys Rev Lett, 2008, 100: 155502

61 GATAN. http://www.gatan.com/products/tem-specimen-holders/ straining-holders

62 Zheng K, Shao R, Wang J, et al. Fabrication of individual carbon nanotubes and their arrays in a transmission electron microscope. Carbon, 2016, 100: 435-440

63 Chisholm C, Bei H, Lowry MB, et al. Dislocation starvation and exhaustion hardening in Mo alloy nanofibers. Acta Mater, 2012, 60: 2258-2264

64 Chen Y, An X, Liao X. Mechanical behaviors of nanowires. Appl Phys Rev, 2017, 4: 031104

65 Wang L, Guan P, Teng J, et al. New twinning route in facecentered cubic nanocrystalline metals. Nat Commun, 2017, 8: 2142

66 Hirth JP, Lothe J, Mura T. Theory of Dislocations (2nd ed.). Berlin: John Wiley \& Sons, 1983.

67 Liu X, Golubov SI, Woo CH, et al. Glide of edge dislocations in tungsten and molybdenum. Mater Sci Eng-A, 2004, 365: 96-100

68 Terentyev D, Osetsky YN, Bacon DJ. Competing processes in reactions between an edge dislocation and dislocation loops in a body-centred cubic metal. Scripta Mater, 2010, 62: 697-700

69 Kuramoto E, Aono Y, Tsutsumi T. Screw dislocation core structure and plastic deformation of BCC Metals. Cryst Res Technol, 2010, 19: 331-339

70 Terentyev DA, Osetsky YN, Bacon DJ. Effects of temperature on structure and mobility of the $<100>$ edge dislocation in bodycentred cubic iron. Acta Mater, 2010, 58: 2477-2482

71 Duesbery MS, Vitek V. Plastic anisotropy in b.c.c. transition metals. Acta Mater, 1998, 46: 1481-1492

72 Weinberger CR, Boyce BL, Battaile CC. Slip planes in bcc transition metals. Int Mater Rev, 2013, 58: 296-314

73 Caillard D. Kinetics of dislocations in pure Fe. Part I. In situ straining experiments at room temperature. Acta Mater, 2010, 58: 3493-3503

74 Caillard D. Kinetics of dislocations in pure Fe. Part II. In situ straining experiments at low temperature. Acta Mater, 2010, 58: 3504-3515

75 Tougou K, Shikata A, Kawase U, et al. In-situ TEM observation of dynamic interaction between dislocation and cavity in BCC metals in tensile deformation. J Nucl Mater, 2015, 465: 843-848

76 Lee HJ, Wirth BD. Molecular dynamics simulation of dislocation-void interactions in BCC Mo. J Nucl Mater, 2009, 386-388: 115-118

77 Noble FW, Hull D. Deformation of single crystals of iron 3\% Silicon. Philos Mag, 1965, 12: 777-796

78 Šesták B, Libovický S. Transition to crystallographic slip on Fe-3\% Si single crystals at 78 K. Acta Metall, 1963, 11: 1190-1191

79 Vitek V. Core structure of screw dislocations in body-centred cubic metals: relation to symmetry and interatomic bonding. Philos Mag, 2004, 84: 415-428

80 Chang R. Screw dislocation core structure in body-centred cubic iron. Philos Mag, 1967, 16: 1021-1029

81 Chaussidon J, Fivel M, Rodney D. The glide of screw dislocations in bcc Fe: Atomistic static and dynamic simulations. Acta Mater, 2006, 54: 3407-3416

82 Grujic K, Hellesø OG. Dielectric microsphere manipulation and chain assembly by counter-propagating waves in a channel waveguide. Opt Express, 2007, 15: 6470-6477

83 Dezerald L, Rodney D, Clouet E, et al. Plastic anisotropy and dislocation trajectory in BCC metals. Nat Commun, 2016, 7: 11695

84 Hoge KG, Mukherjee AK. The temperature and strain rate dependence of the flow stress of tantalum. J Mater Sci, 1977, 12: 1666-1672

85 Kim JY, Jang D, Greer JR. Tensile and compressive behavior of tungsten, molybdenum, tantalum and niobium at the nanoscale. Acta Mater, 2010, 58: 2355-2363

86 Pegel B. Stacking faults on $\{110\}$ planes in the B.C.C. lattice. Phys Stat Sol (b), 1968, 28: 603-609

87 Hirschhorn JS. Stacking faults in the refractory metals and alloys -A review. J Less Common Met, 1963, 5: 493-509

88 Vítek V. Thermally activated motion of screw dislocations in B.C.C. metals. Phys Stat Sol (b), 1966, 18: 687-701

89 Ogata S, Li J, Yip S. Energy landscape of deformation twinning in bcc and fcc metals. Phys Rev B, 2005, 71: 4102

90 Tang Y, Bringa EM, Meyers MA. Inverse Hall-Petch relationship in nanocrystalline tantalum. Mater Sci Eng-A, 2013, 580: 414-426

91 Jeon JB, Lee BJ, Chang YW. Molecular dynamics simulation study of the effect of grain size on the deformation behavior of nanocrystalline body-centered cubic iron. Scripta Mater, 2011, 64: 494-497

92 Ligda J, D'Antuono DS, Taheri ML, et al. Quasi-static tensile and compressive behavior of nanocrystalline tantalum based on miniature specimen testing-Part II: mechanical properties. JOM, 2016, 68: 2839-2846

93 Huang BW, Shang JX, Liu ZH, et al. Atomic simulation of bcc niobium <001> grain boundary under shear deformation. Acta Mater, 2014, 77: 258-268

94 Niu LL, Zhang Y, Shu X, et al. Shear-coupled grain boundary migration assisted by unusual atomic shuffling. Sci Rep, 2016, 6: 23602

95 Yamakov V, Wolf D, Phillpot SR, et al. Deformation-mechanism map for nanocrystalline metals by molecular-dynamics simulation. Nat Mater, 2004, 3: 43-47

96 Wolf D, Yamakov V, Phillpot SR, et al. Deformation of nanocrystalline materials by molecular-dynamics simulation: relationship to experiments? Acta Mater, 2005, 53: 1-40

97 Yamakov V, Wolf D, Phillpot SR, et al. Dislocation processes in the deformation of nanocrystalline aluminium by molecular-dynamics simulation. Nat Mater, 2002, 1: 45-49

98 Lee SW, Cheng YT, Ryu I, et al. Cold-temperature deformation of nano-sized tungsten and niobium as revealed by in-situ nanomechanical experiments. Sci China Technol Sci, 2014, 57: 652662

99 Torrents Abad O, Wheeler JM, Michler J, et al. Temperaturedependent size effects on the strength of Ta and $\mathrm{W}$ micropillars. Acta Mater, 2016, 103: 483-494

100 Huang L, Li QJ, Shan ZW, et al. A new regime for mechanical annealing and strong sample-size strengthening in body centred cubic molybdenum. Nat Commun, 2011, 2: 547

101 Csikor FF, Motz C, Weygand D, et al. Dislocation avalanches, 
strain bursts, and the problem of plastic forming at the micrometer scale. Science, 2007, 318: 251-254

102 Xie KY, Shrestha S, Cao Y, et al. The effect of pre-existing defects on the strength and deformation behavior of $\alpha$-Fe nanopillars. Acta Mater, 2013, 61: 439-452

103 Xie KY, Wang Y, Ni S, et al. Insight into the deformation mechanisms of $\alpha$-Fe at the nanoscale. Scripta Mater, 2011, 65: 10371040

104 El-Awady JA, Wen M, Ghoniem NM. The role of the weakest-link mechanism in controlling the plasticity of micropillars. J Mech Phys Solids, 2009, 57: 32-50

105 Parthasarathy TA, Rao SI, Dimiduk DM, et al. Contribution to size effect of yield strength from the stochastics of dislocation source lengths in finite samples. Scripta Mater, 2007, 56: 313-316

106 Weinberger CR, Cai W. Surface-controlled dislocation multiplication in metal micropillars. Proc Natl Acad Sci USA, 2008, 105: 14304-14307

107 Han SM, Bozorg-Grayeli T, Groves JR, et al. Size effects on strength and plasticity of vanadium nanopillars. Scripta Mater, 2010, 63: 1153-1156

108 Huang R, Li QJ, Wang ZJ, et al. Flow stress in submicron BCC iron single crystals: sample-size-dependent strain-rate sensitivity and rate-dependent size strengthening. Mater Res Lett, 2015, 3: 121-127

109 Kotrechko S, Timoshevskii A, Mikhailovskij I, et al. Atomic mechanisms governing upper limit on the strength of nanosized crystals. Eng Fract Mech, 2015, 150: 184-196

110 Sainath G, Choudhary BK, Jayakumar T. Molecular dynamics simulation studies on the size dependent tensile deformation and fracture behaviour of body centred cubic iron nanowires. Comput Mater Sci, 2015, 104: 76-83

111 Sainath G, Choudhary BK. Molecular dynamics simulations on size dependent tensile deformation behaviour of [110] oriented body centred cubic iron nanowires. Mater Sci Eng-A, 2015, 640: 98-105

112 Taylor G, Christian JW. Experiments on the deformation of niobium single crystals. I. Stress versus strain curves and slip systems in compression and tension. Philos Mag, 1967, 15: 873892

113 Wei Q. Strain rate effects in the ultrafine grain and nanocrystalline regimes-influence on some constitutive responses. J Mater Sci, 2007, 42: 1709-1727

114 Carreker Jr. RP, Hibbard Jr. WR. Tensile deformation of highpurity copper as a function of temperature, strain rate, and grain size. Acta Metall, 1953, 1: 654-663

115 Wang YM, Ma E. Strain hardening, strain rate sensitivity, and ductility of nanostructured metals. Mater Sci Eng-A, 2004, 375377: 46-52

116 Wei Q, Cheng S, Ramesh KT, et al. Effect of nanocrystalline and ultrafine grain sizes on the strain rate sensitivity and activation volume: fcc versus bcc metals. Mater Sci Eng-A, 2004, 381: 71-79

117 Gordon PA, Neeraj T, Li Y, et al. Screw dislocation mobility in BCC metals: the role of the compact core on double-kink nucleation. Model Simul Mater Sci Eng, 2010, 18: 085008

118 Dora JE, Rajnak S. Nucleation of kink pairs and the Peierls' mechanism of plastic deformation. Transactions of the Metallurgical Society of AIME, 1963, 230, 1052-1064

119 Langdon TG. The mechanical properties of superplastic materials. Metall Trans A, 1982, 13: 689-701

120 Van Swygenhoven H, Derlet PM, Frøseth AG. Stacking fault energies and slip in nanocrystalline metals. Nat Mater, 2004, 3: 399-403

121 Liao XZ, Zhou F, Lavernia EJ, et al. Deformation mechanism in nanocrystalline Al: Partial dislocation slip. Appl Phys Lett, 2003, 83: 632-634

122 Li S, Ding X, Li J, et al. High-efficiency mechanical energy storage and retrieval using interfaces in nanowires. Nano Lett, 2010, 10: 1774-1779

123 Li S, Ding X, Deng J, et al. Superelasticity in bcc nanowires by a reversible twinning mechanism. Phys Rev B, 2010, 82: 205435

124 Wang J, Zeng Z, Weinberger CR, et al. In situ atomic-scale observation of twinning-dominated deformation in nanoscale bodycentred cubic tungsten. Nat Mater, 2015, 14: 594-600

125 Marian J, Cai W, Bulatov VV. Dynamic transitions from smooth to rough to twinning in dislocation motion. Nat Mater, 2004, 3: 158-163

126 Chen SR, Gray GT. Constitutive behavior of tantalum and tantalum-tungsten alloys. Metall Mat Trans A, 1996, 27: 2994-3006

127 Voyiadjis GZ, Abed FH. Microstructural based models for bcc and fcc metals with temperature and strain rate dependency. Mech Mater, 2005, 37: 355-378

128 Jia D, Ramesh KT, Ma E. Effects of nanocrystalline and ultrafine grain sizes on constitutive behavior and shear bands in iron. Acta Mater, 2003, 51: 3495-3509

129 Wei Q, Jiao T, Ramesh K, et al. Mechanical behavior and dynamic failure of high-strength ultrafine grained tungsten under uniaxial compression. Acta Mater, 2005, 54: 77-87

130 Malow TR, Koch CC. Mechanical properties, ductility, and grain size of nanocrystalline iron produced by mechanical attrition. Metall Mat Trans A, 1998, 29: 2285-2295

131 Wei Q, Jia D, Ramesh KT, et al. Evolution and microstructure of shear bands in nanostructured Fe. Appl Phys Lett, 2002, 81: $1240-1242$

132 Khan AS, Zhang H, Takacs L. Mechanical response and modeling of fully compacted nanocrystalline iron and copper. Int J Plasticity, 2000, 16: 1459-1476

133 Pan Z, Li Y, Wei Q. Tensile properties of nanocrystalline tantalum from molecular dynamics simulations. Acta Mater, 2008, 56: 3470-3480

134 Wei Q, Pan ZL, Wu XL, et al. Microstructure and mechanical properties at different length scales and strain rates of nanocrystalline tantalum produced by high-pressure torsion. Acta Mater, 2011, 59: 2423-2436

135 Yoo SH, Sudarshan TS, Sethuram K, et al. Consolidation and high strain rate mechanical behavior of nanocrystalline tantalum powder. Nanostructured Mater, 1999, 12: 23-28

136 Wei Q, Jiao T, Mathaudhu SN, et al. Microstructure and mechanical properties of tantalum after equal channel angular extrusion (ECAE). Mater Sci Eng-A, 2003, 358: 266-272

137 Wei Q, Zhang H, Schuster B, et al. Microstructure and mechanical properties of super-strong nanocrystalline tungsten processed by high-pressure torsion. Acta Mater, 2006, 54: 40794089

138 Trelewicz JR, Schuh CA. The Hall-Petch breakdown in nanocrystalline metals: A crossover to glass-like deformation. Acta Mater, 2007, 55: 5948-5958

139 Meyers MA, Mishra A, Benson DJ. Mechanical properties of nanocrystalline materials. Prog Mater Sci, 2006, 51: 427-556

140 Carlton CE, Ferreira PJ. What is behind the inverse Hall-Petch effect in nanocrystalline materials? Acta Mater, 2007, 55: 3749- 
3756

141 Farkas D, Hyde B. Improving the ductility of nanocrystalline bcc metals. Nano Lett, 2005, 5: 2403-2407

142 Latapie A, Farkas D. Molecular dynamics investigation of the fracture behavior of nanocrystalline a-Fe. Phys Rev B, 2004, 69: 460-461

143 Zhang Y, Millett PC, Tonks M, et al. Deformation twins in nanocrystalline body-centered cubic Mo as predicted by molecular dynamics simulations. Acta Mater, 2012, 60: 6421-6428

144 Zhang Y, Millett PC, Tonks M, et al. Deformation-twin-induced grain boundary failure. Scripta Mater, 2012, 66: 117-120

145 Cheng GM, Jian WW, Xu WZ, et al. Grain size effect on de- formation mechanisms of nanocrystalline bcc metals. Mater Res Lett, 2013, 1: 26-31

Acknowledgements This work was supported by the Key Project of the National Natural Science Foundation of China (11234011). The authors thank the support and encouragement of Prof. Xiaodong Han and Prof. Lihua Wang.

Author contributions Liao X initiated and guided the whole work. Lu $\mathrm{Y}$ and Shu $\mathrm{X}$ wrote the manuscript, surveyed the literatures and designed figures. All the authors contributed to the general discussion.

Conflict of interest The authors declare no conflict of interest.

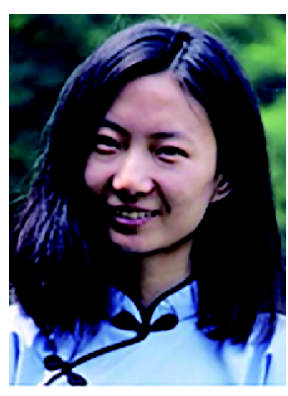

Yan Lu is a PhD candidate in Beijing Key Laboratory and the Institute of Microstructure and Properties of Advanced Materials, Beijing University of Technology. Her current research is focused on in-situ straining electron microscopy investigation of the deformation behaviors of nanocrystalline body-centered cubic metals at the atomic scale.
Xinyu Shu received his Master degree from Beijing University of Technology. He is now a PhD candidate in the Department of Material Science and Engineering, National University of Singapore. His current research interest is spintronics, including spin-orbit torque induced switching and ferromagnetic resonance.

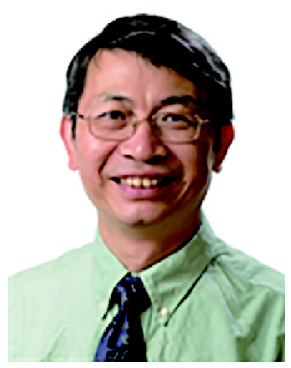

Xiaozhou Liao received his PhD in physics from the University of Sydney. He is currently a professor in the School of Aerospace, Mechanical and Mechatronic Engineering at the University. He uses advanced electron microscopy techniques to examine materials at the atomic scale, laying the groundwork for the design of superior materials for applications including in the semiconductor, automobile and aviation industries.

\title{
体心立方金属及合金的高力学性能的尺寸效应
}

\author{
卢艳 ${ }^{1}$, 舒新愉 ${ }^{1,2}$, 廖晓 舟 $^{{ }^{*}}$
}

摘要 体心立方 $(\mathrm{BCC})$ 金属在亚微米和纳米尺度表现出不同于块体粗晶尺度的力学性能, 包括高屈服强度和大延展性. 这些突出性能使其 不仅可用于微米、纳米甚至原子尺度的器件, 而且可作为结构材料的组成部分发挥重要作用. 导致小尺度BCC金属这种不同寻常的力学 性能的一个重要原因就是它们的尺寸, 降低单晶金属的尺寸和多晶金属的晶粒度可以显著增加强度. 在过去十年, 小尺度BCC金属塑性变 形行为的研究取得了大幅进展. 本综述全面、系统地总结了小尺度BCC金属塑性变形尺寸效应的塑性机制理论, 并综合介绍了研究小尺 寸样品塑性变形的原位方法和技术. 